\title{
Rapid Mixed Layer Deepening by the Combination of Langmuir and Shear Instabilities: A Case Study
}

\author{
TOBIAS KUKULKA* \\ Department of Physical Oceanography, and Department of Applied Ocean Physics and Engineering, Woods Hole Oceanographic \\ Institution, Woods Hole, Massachusetts
}

Albert J. PluedDemanN

Department of Physical Oceanography, Woods Hole Oceanographic Institution, Woods Hole, Massachusetts

JOHN H. TROWBRIDGE

Department of Applied Ocean Physics and Engineering, Woods Hole Oceanographic Institution, Woods Hole, Massachusetts

Peter P. Sullivan

National Center for Atmospheric Research, ${ }^{+}$Boulder, Colorado

(Manuscript received 23 November 2009, in final form 21 June 2010)

\begin{abstract}
Langmuir circulation (LC) is a turbulent upper-ocean process driven by wind and surface waves that contributes significantly to the transport of momentum, heat, and mass in the oceanic surface layer. The authors have previously performed a direct comparison of large-eddy simulations and observations of the upper-ocean response to a wind event with rapid mixed layer deepening. The evolution of simulated crosswind velocity variance and spatial scales, as well as mixed layer deepening, was only consistent with observations if LC effects are included in the model. Based on an analysis of these validated simulations, in this study the fundamental differences in mixing between purely shear-driven turbulence and turbulence with LC are identified. In the former case, turbulent kinetic energy (TKE) production due to shear instabilities is largest near the surface, gradually decreasing to zero near the base of the mixed layer. This stands in contrast to the LC case in which at middepth range TKE production can be dominated by Stokes drift shear. Furthermore, the Eulerian mean vertical shear peaks near the base of the mixed layer so that TKE production by mean shear flow is elevated there. LC transports horizontal momentum efficiently downward leading to an along-wind velocity jet below LC downwelling regions at the base of the mixed layer. Locally enhanced vertical shear instabilities as a result of this jet efficiently erode the thermocline. In turn, enhanced breaking internal waves inject cold deep water into the mixed layer, where LC currents transport temperature perturbation advectively. Thus, LC and locally generated shear instabilities work intimately together to facilitate strongly the mixed layer deepening process.
\end{abstract}

\section{Introduction}

Upper-ocean turbulence plays a key role in weather and climate systems because it couples the ocean and

* Current affiliation: University of Delaware, Newark, Delaware.

+ The National Center for Atmospheric Research is sponsored by the National Science Foundation.

Corresponding author address: Tobias Kukulka, University of Delaware, Newark, DE 19716.

E-mail: kukulka@udel.edu atmosphere through air-sea fluxes of heat, momentum, and mass. Breaking and nonbreaking ocean surface waves may control oceanic turbulence near the air-sea interface (Leibovich 1983; Melville 1996; Thorpe 2004). Breaking ocean surface waves transfer some of their energy to subsurface turbulent kinetic energy (Craig and Banner 1994; Terray et al. 1996; Melville 1996). The resulting enhanced turbulence intensities and dissipation rates, in turn, are expected to contribute significantly in mixing very near the surface. Nonbreaking ocean surface waves influence upper-ocean turbulence 
because greater below-crest and smaller below-trough wave orbital speeds induce a residual circulation (Stokes drift) that tilts vertical vorticity into the direction of wave propagation (Craik and Leibovich 1976). This vortex tilting interacts with sheared surface currents to form wind-aligned roll vortices, called Langmuir circulation (LC) (Langmuir 1938).

The goal of this paper is to investigate the turbulent processes and mechanisms contributing to rapid mixed layer deepening, which has been observed in open oceans (Smith 1992; Plueddemann et al. 1996). Although, generally, both breaking waves and LC are integral to upperocean mixing, this article focuses only on the contribution of LC to mixed layer dynamics. Our assumption-that mixing processes near the base of the mixed layer (with an average depth of about $20 \mathrm{~m}$ in this study) do not depend to first order on the near-surface layer dynamics that is significantly influenced by breaking waves-is supported by previous studies. Observed enhanced turbulent kinetic energy (TKE) dissipation rates, often attributed to breaking wave TKE fluxes, extend to a depth on the order of one significant wave height (Terray et al. 1996), which is for our study less than $3 \mathrm{~m}$. Furthermore, the good agreement between observations and model results with only LC effects of the same mixed layer deepening event examined here indicates that neglecting breaking waves is a viable approach for our study (Kukulka et al. 2009).

Observing and modeling LC has been an intriguing research problem for over seven decades. Elaborate field observations revealed many distinguished LC features, such as their temporal evolution with wind and wave conditions (Weller and Price 1988; Smith 1992; Farmer and Li 1995; Plueddemann et al. 1996; D'Asaro and Dairiki 1997). Modeling approaches usually rely on the wave-averaged Navier-Stokes equations, based on the systematic mathematical theory by Craik and Leibovich (1976) and Leibovich (1983). Numerical simulations, which resolve the flux and energy carrying eddies in an approach called "large-eddy simulations" (LES) (Skyllingstad and Denbo 1995; McWilliams et al. 1997; Li et al. 2005; Tejada-Martínez and Grosch 2007; Polton and Belcher 2007; Harcourt and D'Asaro 2008; Grant and Belcher 2009), yield important insights regarding the dynamics and structure of LC. Many LES results agree at least qualitatively with observations, but a thorough comparison of observations and simulations for a wide range of wind and wave conditions remains an outstanding research goal.

Previously, we have validated LES solutions based on observations from the North Pacific Ocean during a 3-h wind event (Kukulka et al. 2009). We found that the simulations of the evolution of subsurface crosswind velocity wavenumber spectra are only consistent with observations if LC effects are included in the model. Furthermore, model results indicate that the observed rapid mixed layer deepening is attributed to LC. Taking advantage of the four-dimensional LES dataset from our previous study validated by comparison with observations, the objective of this paper is to dissect the physical mechanisms behind the enhanced mixed layer deepening in the presence of LC.

Generally, both observations and numerical simulations suggest that LC can significantly enhance mixed layer deepening. In particular, LES studies indicate that the buoyancy entrainment flux may be greatly enhanced by LC (e.g., McWilliams et al. 1997; Grant and Belcher 2009). Despite this progress, the detailed role of LC processes contributing to entrainment at the base of the mixed layer remains incompletely understood (see, e.g., discussion by Thorpe 2004). For example, from LES results Skyllingstad et al. (2000) conclude that LC effects were mostly confined to the initial mixed layer deepening and were, otherwise, a second-order effect compared to resonant wind forcing. Observations from Weller and Price (1988) indicate that LC play an important role in maintaining a shallow diurnal mixed layer but do not directly contribute to mixing processes near the base of the mixed layer between 40-m and 60-m depth. Based on a two-dimensional model with preexisting stratification, $\mathrm{Li}$ and Garrett (1997) suggest two important mixed layer deepening mechanisms. The first mechanism involves an engulfment process in which thermocline water is advected by LC into the mixed layer. For the second mechanism, the LC locally enhances shear instability at the base if the mixed layer. Interestingly, there is little documented evidence for either mechanism based on observations or LES. We will examine in detail the turbulence characteristics during a mixed layer deepening event and highlight a mechanism that involves the tight coupling of LC and shear instabilities at the base of the mixed layer.

\section{Observations and simulations of the upper-ocean response to a wind event}

The methodology presented here is based on work by Kukulka et al. (2009) in which we compared observations and numerical simulations of the upper-ocean response to a wind event. We will here reiterate the methodological approach following closely Kukulka et al. (2009), but provide additional details.

\section{a. Observations}

The Surface Waves Processes Program (SWAPP) was conducted in the Pacific Ocean about $550 \mathrm{~km}$ west off 
the California coast during February and March 1990 (Smith 1992; Plueddemann et al. 1996). The SWAPP dataset provides comprehensive measurements of (i) ocean surface wave fields; (ii) heat and momentum airsea fluxes; (iii) vertical profiles of temperature, salinity, and currents; as well as (iv) unique subsurface turbulence estimates from "LC detectors" (discussed below). The experimental setting at times closely resembles the idealized open ocean conditions often assumed in LES studies. We focus our analysis on a single 3 -h wind event from 0700 to 1000 PST 4 March, when wind and waves were approximately unidirectional and aligned. The wind speed at 10-m height increased from $U_{10}=8 \mathrm{~m} \mathrm{~s}^{-1}$ at 0700 to $13 \mathrm{~m} \mathrm{~s}^{-1}$ at 0800 PSTand then weakened again to about $10 \mathrm{~m} \mathrm{~s}^{-1}$. The significant wave height weakly developed from $H_{s}=2.6$ to $2.9 \mathrm{~m}$. The surface heat fluxes changed from cooling between 60 and $90 \mathrm{~W} \mathrm{~m}^{-2}$ to warming around 0900 PST and approached a value of $200 \mathrm{~W} \mathrm{~m}^{-2}$ at 1000 PST. Since the magnitude of the Monin-Obukhov length exceeds $100 \mathrm{~m}$, which is much larger than the $O(10 \mathrm{~m})$-deep mixed layer, buoyancy effects are likely to play a secondary role in the upperocean turbulence dynamics. The Hoenniker number, which describes buoyancy forces due to surface heat fluxes relative to the Craik-Leibovich (CL) vortex force, is generally less than 0.1 , indicating that wave forcing is dominant over buoyancy forcing ( $\mathrm{Li}$ et al. 2005). From 0730 to 0900 PST observations indicate that larger-scale advective processes played a relatively small role in the near-surface ocean temperature evolution between depths of 7 and $27 \mathrm{~m}$ (Smith 1992).

The LC detectors consist of special purpose acoustic instruments that measure horizontal velocities of surface trapped bubbles over a horizontal range of a few hundred meters (Smith 1989; Zedel and Farmer 1991). In the presence of LC, sonar beams oriented perpendicular to the wind direction (crosswind) detect horizontal bands due to velocity convergence zones of coherent surface LC structures. To compare measurements with model results, it is necessary to understand the vertical extent of these surface measurements. The near-surface bubble distribution decreases roughly exponentially in the vertical with a decay scale around 1.0$1.5 \mathrm{~m}$ and confines the vertical extent of the measurement volume to about $3 \mathrm{~m}$, depending on wind and wave conditions. These measurements are invaluable in diagnosing model results as well as in setting up the model initial, boundary, and forcing conditions.

\section{b. Simulations}

We adopt the filtered (LES) CL equations as described by McWilliams et al. (1997). The governing momentum equation is

$$
\begin{aligned}
\frac{\partial u_{i}}{\partial t} & +u_{j} \frac{\partial u_{i}}{\partial x_{j}}+\epsilon_{i k m} f_{k}\left(u_{m}+u_{s, m}\right) \\
= & -\frac{\partial \pi}{\partial x_{i}}+\frac{\rho}{\rho_{0}} g_{i}+\epsilon_{i k m} u_{s, k} \omega_{m}+\text { SGS }
\end{aligned}
$$

where $t$ denotes time; the index $i=1,2,3$ symbolizes the along-wind, crosswind, and vertical directions, respectively; $\left(x_{1}, x_{2}, x_{3}\right)=(x, y, z)$ are the spatial coordinates; $\left(u_{1}, u_{2}, u_{3}\right)=(u, v, w)$ is the velocity vector; $\left(f_{1}, f_{2}, f_{3}\right)=$ $(0,0, f)$ is the Coriolis vector with the Coriolis parameter $f=10^{-4} \mathrm{~s}^{-1} ;\left(g_{1}, g_{2}, g_{3}\right)=(0,0,-g)$ is the earth's acceleration vector with $g=9.81 \mathrm{~m} \mathrm{~s}^{-2} ;\left(u_{s, 1}, u_{s, 2}, u_{s, 3}\right)=$ $\left(u_{s}, 0,0\right)$ is the Stokes drift vector; $\pi=p / \rho_{0}+1 / 2\left[\left(u_{i}+\right.\right.$ $\left.\left.u_{s, i}\right)\left(u_{i}+u_{s, i}\right)-u_{i} u_{i}\right]$ is a generalized pressure in which $p$ is the pressure and $\rho$ is the density, $\rho_{0}$ is a constant reference density; $\omega_{i}=\epsilon_{i k m}\left(\partial / \partial x_{k}\right) u_{m}$ is the relative vorticity; $\epsilon_{i k m}$ is the Levi-Civita (permutation) tensor; and SGS symbolizes subgrid-scale terms (see appendix B for details). The CL momentum equations capture LC dynamics by a vortex force that involves the Stokes drift [third term on the rhs in (1)]. If the Stokes drift is set to zero ("no LC" case), the LES model simply solves the spatially averaged Navier-Stokes equations without wave forcing but still captures shear and buoyancy instabilities.

The governing density equation and continuity equations are

$$
\begin{aligned}
\frac{\partial \rho}{\partial t}+\left(u_{j}+u_{s, j}\right) \frac{\partial \rho}{\partial x_{j}} & =\text { SGS and } \\
\frac{\partial u_{j}}{\partial x_{j}} & =0,
\end{aligned}
$$

respectively. The density and temperature are linearly related by

$$
\alpha=-\frac{1}{\rho} \frac{\partial \rho}{\partial T}
$$

with the constant coefficient of thermal expansion $\alpha=$ $2 \times 10^{-4} \mathrm{~K}^{-1}$.

For the analysis below, we note that any resolved variable $q$ can be furthermore decomposed into its horizontal average $\langle q\rangle$ and its deviation from the horizontal average $q^{\prime}$,

$$
q=\langle q\rangle+q^{\prime} .
$$

Details on the LES model are described in appendix B. At the ocean surface, time varying heat and momentum fluxes are specified based on the observations. An internal gravity wave radiation condition is imposed at the bottom of the computational domain. 
The initial fields of velocity, temperature, and SGS energy were obtained in two stages so as to obtain fully developed LES turbulence fields consistent with the observations (for details, see Kukulka et al. 2009). Temperature profile data, which are used to set up the initial conditions, were obtained from vector-measuring current meters (VMCMs) and conductivity-temperature depth (CTD) instruments. For initialization, temperature profiles from the VMCM data are used because these data are closer to the surface with the shallowest measurement at $z=-2.25 \mathrm{~m}$. For comparison of observed and simulated temperature profiles (discussed below), CTD data are used because these are sampled with higher vertical resolution. The stratification across the MLD (e.g., from 6 to $12 \mathrm{~m}$ ) is comparable for the VMCM and CTD profiles (e.g., $0.016^{\circ}$ and $0.014^{\circ} \mathrm{C} \mathrm{m}^{-1}$, respectively, at $0630 \mathrm{PST}$ ) although the more highly resolved CTD profile has stronger local gradients. The temperature profile measured at 0630 PST was imposed and the simulation was forced for $1 \mathrm{~h}$ with air-sea flux data obtained from 0530 to 0630 PST (roughly two eddy turnover periods; strong temperature gradients were at about $8-\mathrm{m}$ depth) so that the turbulent fields could adjust to the observed temperature profile. Based on these results the temperature field was reinitialized with the measured 0630 PST profile and observed fields from 0630 to 1000 PST were simulated by imposing the observed surface fluxes and wave forcing in the LC case.

The time-dependent Stokes drift in the CL equations was estimated based on a monochromatic surface wave whose amplitude is consistent with the significant wave height obtained from observed time-dependent wave height spectra. The frequency of the monochromatic wave is determined by matching its Stokes drift to the Stokes drift at $z=-2.25 \mathrm{~m}$ that has been calculated previously from integration of the observed wave spectrum. The same approach was used by Kukulka et al. (2009). The resulting wave periods range between 7 and $8 \mathrm{~s}$, which is within the range of typically observed wind wave peak periods (4-10 s) but smaller than common swell periods of 12-14 s. Thus, our frequency estimate likely captures more accurately the contributions of shorter waves to the Stokes drift than one based on a monochromatic wave at the peak frequency of the wave height spectrum. It would have been more straightforward to use a Stokes drift profile estimated directly from integration of observed surface wave spectra, but the spectral data were not readily available to us (whereas the computed Stokes drift at $2.25 \mathrm{~m}$ was). Sensitivity tests using Stokes drift profiles computed by Smith (cf. Smith and Bullard 1995) showed minor differences in the strength of the Langmuir circulation during the event but no qualitative differences that would impact the results or conclusions presented here.
The turbulent Langmuir number $\mathrm{La}_{t}=\left[u_{*} / u_{s}(z=0)\right]^{1 / 2}$ (McWilliams et al. 1997) is between $\mathrm{La}_{t}=0.3$ and 0.6 during the wind event. According to the turbulence regime diagram from Li et al. (2005), the turbulence investigated here is mainly driven by surface waves through LC instabilities.

Our default model domain spans a $200 \mathrm{~m} \times 200 \mathrm{~m}$ horizontal and 60-m-deep ocean volume with $256 \times 256$ horizontal and 150 vertical grid points; that is, the grid resolution is $\Delta x \approx 0.78 \mathrm{~m}$ in the horizontal and $\Delta z=$ $0.4 \mathrm{~m}$ in the vertical. A relatively high grid resolution is particularly important without LC to capture small-scale stratified turbulence, which results from shear instabilities and accomplishes mixing near the thermocline (Skyllingstad et al. 2000; Beare et al. 2006; Ivey et al. 2008). Our default domain and grid resolves the flux and energy carrying eddies. During rapid mixed layer deepening, the Ozmidov length scale $L_{O}$ near the mixed layer base is about $0.8 \mathrm{~m}$ without LC and $1.3 \mathrm{~m}$ with LC if $L_{O}=\sqrt{\langle\epsilon\rangle /\langle N\rangle^{3}}$, and $L_{O}=1.4$ and $2.7 \mathrm{~m}$ without and with LC, respectively, if $L_{O}=\sqrt{\left\langle w^{2}\right\rangle} /\langle N\rangle$, suggesting that our LES with $\Delta z=0.4 \mathrm{~m}$ is close to the resolution limit in the no-LC case. Without LC (the more challenging simulation, as eddies are smaller) we also ran the experiment on a $100 \mathrm{~m} \times 100 \mathrm{~m} \times 45 \mathrm{~m}$ domain with $250 \times 250 \times 150$ and $128 \times 128 \times 75$ grid points. These and other sensitivity experiments with different domain and grid sizes indicate that a higher resolution and a larger domain does not significantly change the results presented here.

\section{Results}

\section{a. Evaluation of LES solutions}

Large-eddy-simulation solutions are based on a relatively complex code and consist of lengthy computations with voluminous output. Thus, before diagnosing dynamical processes based on the LES it is important to confirm the fidelity of the model. To this end, we first report two consistency checks that confirm the solutions are consistent with the governing equations. To confirm that the LES adequately represents the phenomena of interest, we supplement previous comparisons (Kukulka et al. 2009) with two qualitative comparisons with SWAPP observations.

\section{1) CONSISTEnCY CHECKS}

To ensure that the model implementation is consistent with known dynamical properties of the flow field, we will examine 1) the role of the Craik-Leibovich force and time-varying wind stress in the vertically integrated transport and 2) the turbulent buoyancy flux and the rate 
of change of potential energy. The vertically integrated transport can be expressed in a closed-form analytic solution as (see appendix A and also Gnanadesikan and Weller 1995; McWilliams et al. 1997; McWilliams and Restrepo 1999; Polton and Belcher 2007)

$$
\mathbf{T}(t)=e^{-i f t}\left\{\int_{0}^{t} e^{i f t^{\prime}}\left[\tau\left(t^{\prime}\right)-i f T_{s}\left(t^{\prime}\right)\right] d t^{\prime}+\mathbf{T}_{0}\right\},
$$

where $\tau$ is the wind stress divided by water density; $T_{s}$ is the Stokes drift transport; $\mathbf{T}=T_{x}+i T_{y}$ with $i=\sqrt{-1}$ in which $T_{x}$ and $T_{y}$ are the along-wind and crosswind transports, respectively [i.e., $\left(T_{x}, T_{y}, T_{s}\right)=\int_{-H}^{0}(\langle u\rangle,\langle v\rangle$, $\left.\left.u_{s}\right) d z\right] ; H$ is the ocean depth; and $\mathbf{T}_{0}$ denotes the initial transport. With $T_{s}=\mathbf{T}_{0}=0$ and $\tau=$ const (6) simplifies to the familiar solution (see, e.g., Gill 1982, p. 322)

$$
\mathbf{T}(t)=-i \frac{\tau}{f}\left(1-e^{-i f t}\right)
$$

where the transport consists of an Ekman transport and inertial oscillation. The solution (6) generally also contains an inertial oscillation with amplitude and phase that satisfies the initial condition. Time variation in the (unidirectional) wind stress and Stokes drift furthermore elicit rotating currents with along-wind and crosswind components in the model response. The solution (6) agrees well with the transport obtained from the LES solutions (Fig. 1).

Turbulent advection of warmer (cooler) water downward (upward) results in buoyancy fluxes and vertical mixing of temperature. Therefore, turbulent kinetic energy that accomplishes temperature mixing near the thermocline is converted to potential energy to elevate the systems total potential energy. This conversion is related to the overall mixed layer deepening. An expression for the total potential energy can be derived from the density transport equation (note that $w=w^{\prime}$ ):

$$
\frac{\partial\langle\rho\rangle}{\partial t}=-\frac{\partial\left\langle w \rho^{\prime}\right\rangle}{\partial z}+\text { SGS }
$$

Multiplying (8) by $z g$, evoking the product rule, integrating over the whole water column, and finally neglecting density fluxes through bottom and surface boundaries results in

$$
\frac{d}{d t} \int_{-H}^{0} z g\langle\rho\rangle d z=\int_{-H}^{0} g\left\langle w \rho^{\prime}\right\rangle d z+\mathrm{SGS}
$$

where the left-hand side is the rate of change of total potential energy per unit surface area and the first term on the right-hand side is the vertically integrated resolved

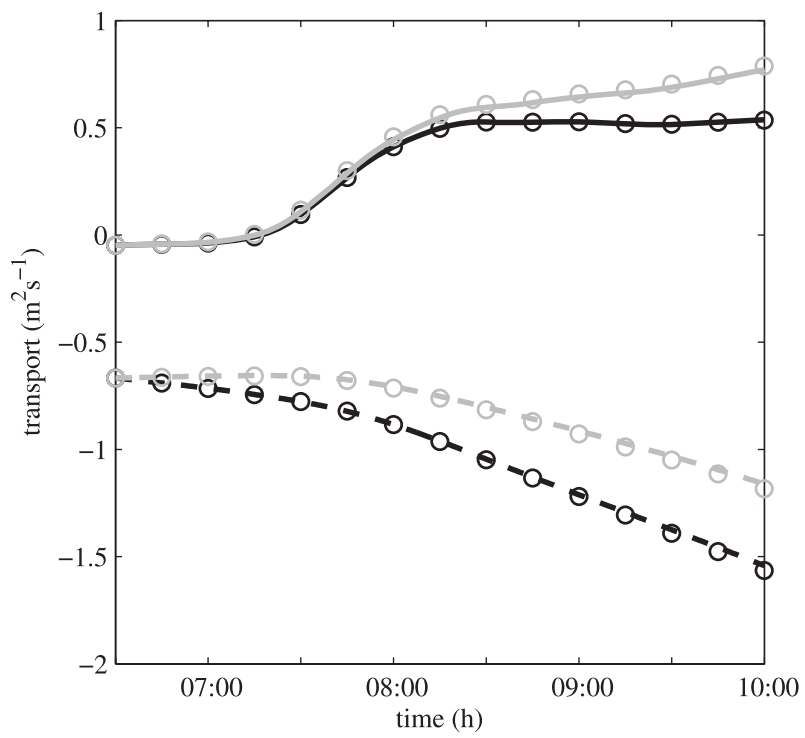

FIG. 1. Along-wind transport $T_{x}$ (solid lines) and crosswind transport $T_{y}$ (dashed lines) obtained from LES solutions with (black) and without (gray) LC. The corresponding solution (6) in circles agrees well with the transport obtained from the LES.

turbulent buoyancy flux. Figure 2 indicates that the change of potential energy is mainly determined by the resolved vertically integrated buoyancy fluxes. Therefore, the dominant turbulent mixing processes are resolved in our LES and do not strongly depend on details of the SGS parameterizations. These results provide an important additional test and further confidence in the complex LES solutions, on which our turbulence analysis in the following subsections will be based.

\section{2) COMPARISON WITH OBSERVATIONS}

Previously, we confirmed that the LES accurately represented evolution of the surface boundary layer for the 4 March SWAPP wind event based on observations of temperature and crosswind velocity variations (Kukulka et al. 2009). We found that the modeled evolution of crosswind velocity variance and spatial scales as well as mixed layer deepening are only consistent with observations if the LC effects are included in the model. Two additional qualitative comparisons are presented here.

First, to investigate further the relation between the mixed layer depth $h$ and LC scales, we estimate a dominant Langmuir cell size $l_{\mathrm{LC}}$ for the major growth period from 0700 to 0900 PST (Fig. 3). For straightforward comparison, we follow here the method by Smith and determine $l_{\text {LC }}$ based on the spectrum-squared weighted wavenumber (Smith 1992). Consistent with Kukulka et al. (2009), $h$ and $l_{\mathrm{LC}}$ increase concurrently in the observed and LC case, albeit at different rates. We find 


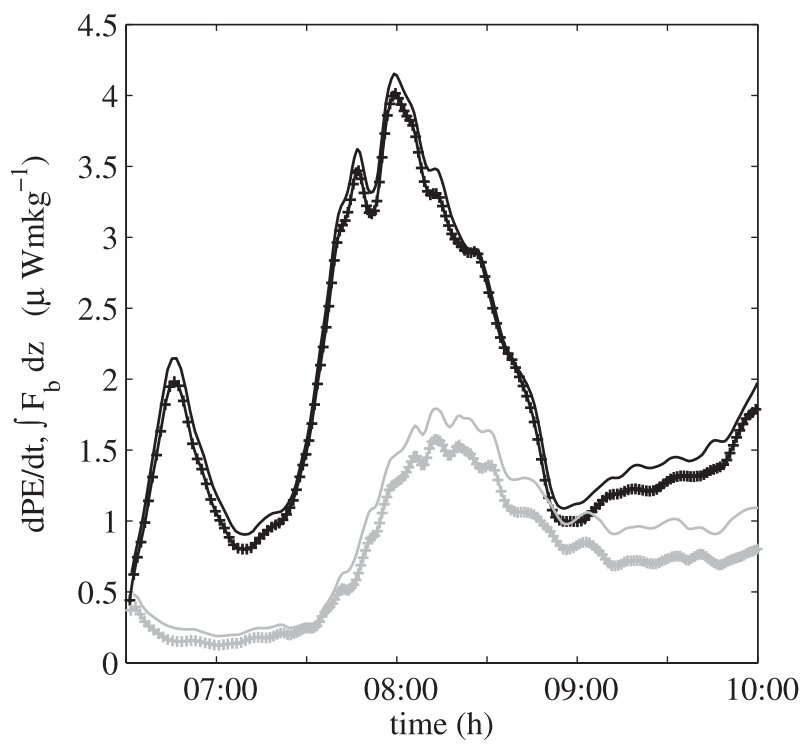

FIG. 2. The resolved vertically integrated buoyancy flux $F_{b}$ [first rhs term in (9) divided by density (solid line with pluses)] is the dominant contribution to the rate of change of potential energy PE [lhs term in (9) divided by density (solid line)]. LES solutions with (without) LC are in black (gray).

an approximate proportional relationship of $l_{\mathrm{LC}} \propto 1.7 \mathrm{~h}$ for both observations and the LC case of the model, where the proportionality constant has been chosen subjectively.

Second, we compare directly the high-resolution density profiles from the CTD system with simulated density profiles (Fig. 4). Note that, unlike the comparison in Kukulka et al. (2009), the simulated profiles are not horizontally averaged, preserving important spatial details. In both observations and simulations, enhanced mixing can be observed from about 0740 PST. In simulations with the LC the mixed layer deepens more rapidly. The simulations with the LC also capture the intermittent presence of thermocline water in the mixed layer, described as "floating blobs of denser water" (Smith 1992). Furthermore, with the LC the modeled horizontal and vertical scales of cold and warm water intrusions are similar to the observed ones, providing evidence that the numerical solutions realistically resolve critical details of the mixed layer system.

\section{b. Instantaneous horizontally averaged profiles of velocity and temperature}

Here we focus on instantaneous horizontally averaged profiles of temperature and velocity and the implications of such profiles for shear instabilities. The profiles in Fig. 5 are representative of times with maximum mixed layer deepening (at 0800 PST, see Fig. 3).

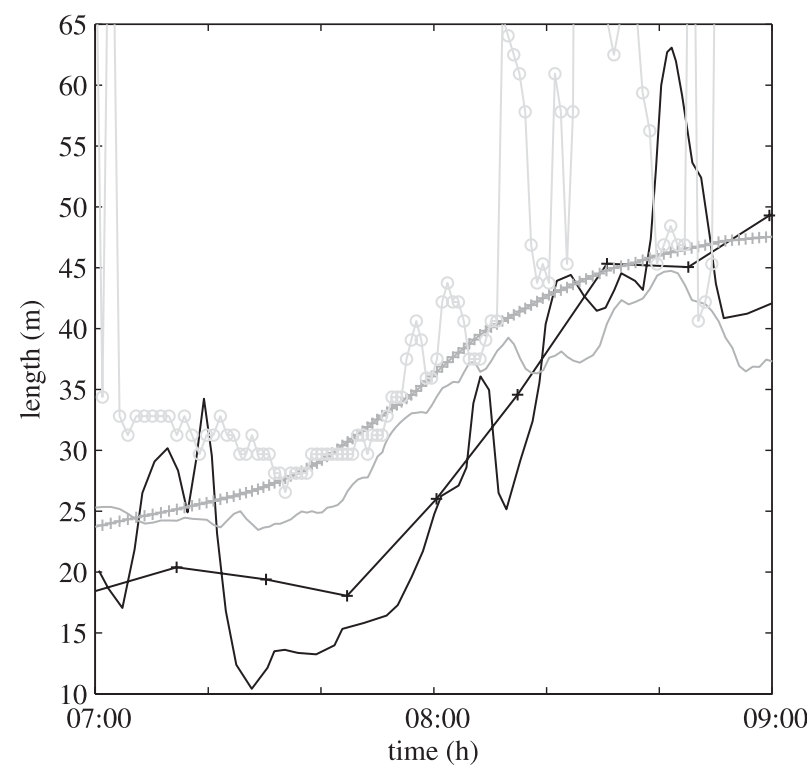

FIG. 3. Comparison of observed (black) and simulated (gray) length scales during LC development. A multiple of the mixed layer depth, $1.7 h$ (lines with pluses), is compared with the dominant LC scale estimate $l_{\text {LC }}$ (lines without symbols) determined from wavenumber spectra following Smith (1992). Light gray line with circles indicates an independent estimate of the LC width $l_{c}$ based on the conditional averages discussed in section $3 \mathrm{e}$.

\section{1) No-LC SOLUTION}

Without LC the along-wind and crosswind velocities gradually decrease from the surface to the base of the mixed layer, which is located at roughly $14 \mathrm{~m}$ (gray lines, left panel in Fig. 5). Enhanced along-wind near-surface gradients in the upper $5 \mathrm{~m}$ indicate relatively weak nearsurface mixing due to smaller eddies and significant vertical transport of horizontal momentum due to the wind stress. Noticeably, a well-mixed momentum layer is absent in the no-LC solution. The temperature profile (gray line, center panel in Fig. 5), on the other hand, does show a relatively well-mixed region in the upper $10 \mathrm{~m}$, where shear instabilities drive mixing and temperature fluxes are relatively weak. Destabilizing shear flow competes with stabilizing stratification, which increases gradually from the surface toward the base of the mixed layer. This competition can be described by the gradient Richardson number:

$$
\mathrm{Ri}=-\frac{g}{\langle\rho\rangle} \frac{d\langle\rho\rangle}{d z}\left[\left(\frac{d\langle u\rangle}{d z}\right)^{2}+\left(\frac{d\langle v\rangle}{d z}\right)^{2}\right]^{-1}
$$

where horizontal averages are indicated by angle brackets. Consistent with the temperature and velocity profiles, Ri increases with depth and exceeds a critical value of about $\mathrm{Ri}=0.25$ in the vicinity of the mixed 


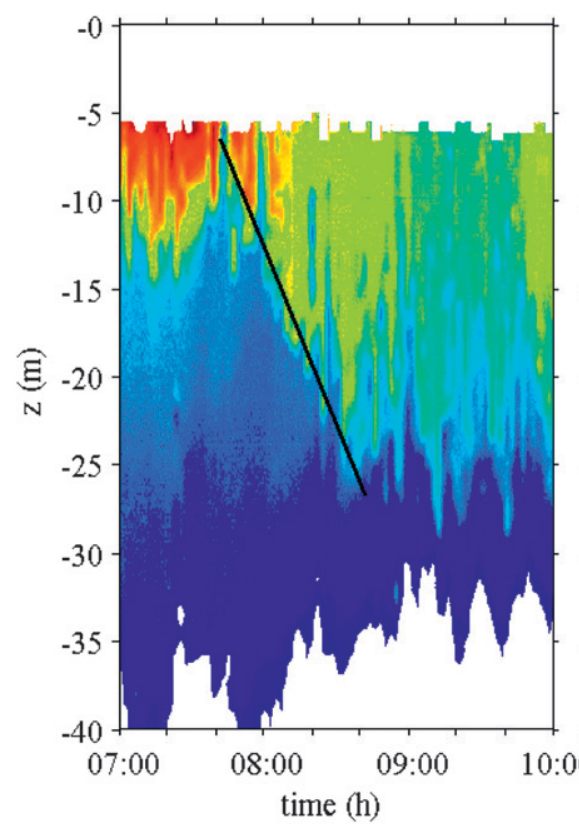

$07: 00$
$07: 00$

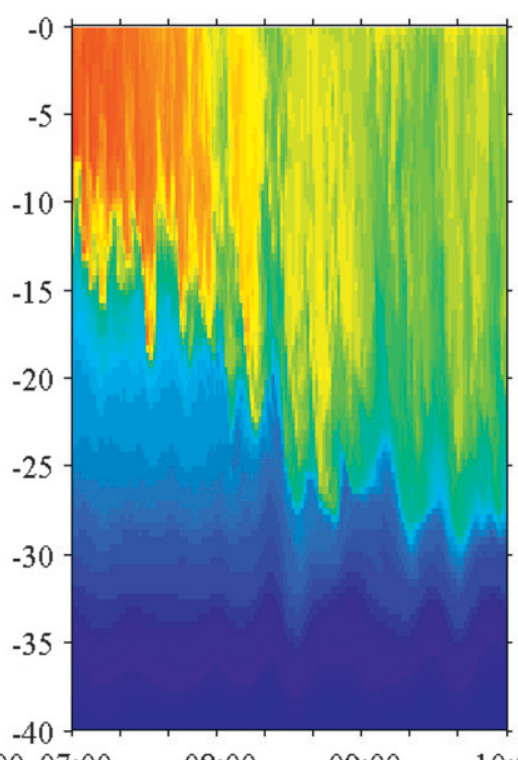

08:00 09:00 10:00
time $(\mathrm{h})$
$\sigma_{\mathrm{T}}\left(\mathrm{kg} \mathrm{m}^{-3}\right)$

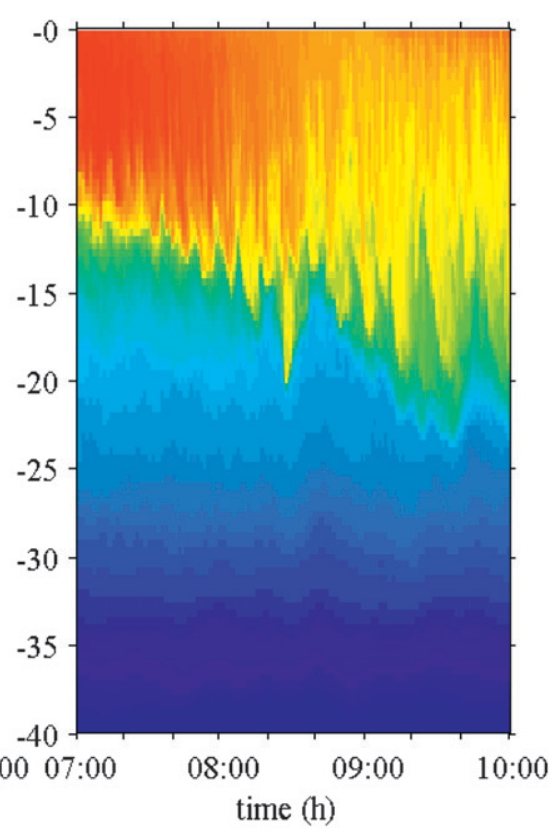

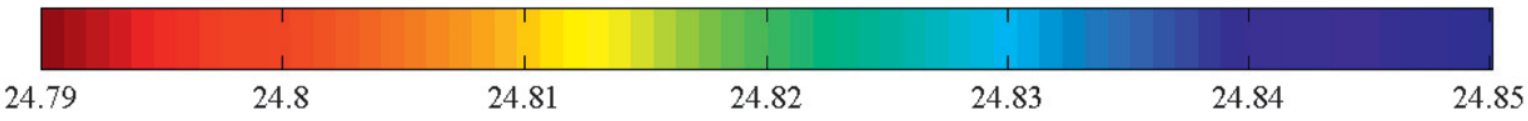

FIG. 4. Comparison of density profiles ( $\sigma_{T}$, arbitrary constant density offset): (left) observed (from Smith 1992) simulated (middle) with LC and (right) without LC. The black line indicates a deepening rate of $20 \mathrm{~m} \mathrm{~s}^{-1}$.

layer base (gray line, right panel of Fig. 5). In summary, horizontally averaged profiles based on LES solutions without LC suggest that shear instabilities drive turbulence and mixing, which gradually decrease in intensity from the surface to the base of the mixed layer.

\section{2) LC SOLUTION}

Unlike the no-LC solution, enhanced mixing due to LC results in a well-mixed momentum layer between depths of $\sim 2$ and $13 \mathrm{~m}$ (black lines, left panel of Fig. 5). Similar to the no-LC case, strong near-surface gradients of along-wind velocity are formed to flux downward the horizontal momentum imposed by the wind stress. In remarkable contrast to the no-LC solution, efficient momentum transport by LC leads to a second depth region of enhanced shear flow located close to the base of the mixed layer between $\sim 16$ and $20 \mathrm{~m}$. This shear must overcome the stabilizing temperature gradients of the thermocline so as to contribute to the turbulence production and to erode the base of the mixed layer. The temperature profile is relatively homogeneous over the first 12-m depth, below which temperature gradually decreases toward the base of the mixed layer (black line, middle panel of Fig. 5). The resulting Ri profile contains a local minimum near the base of the mixed layer (black line, right panel of Fig. 5). We conclude that one effect of the LC is to rapidly transport momentum downward, producing a well-mixed momentum layer near the surface and increasing the likelihood of shear instability near the mixed layer base. This could provide an effective mechanism for eroding the thermocline and mixed layer deepening.

\section{c. Horizontally averaged TKE budgets for resolved scales}

Without LC, the Ri profiles suggest that turbulent kinetic energy extraction from the Eulerian mean flow via shear instabilities is elevated close to the surface and vanishes toward the base of the mixed layer. With LC, on the other hand, we anticipate that TKE shear production has a second local maximum near $h$. The horizontally averaged TKE budget, can be expressed as (e.g., Skyllingstad et al. 2000)

$$
\begin{aligned}
\frac{\partial\left\langle u_{i}^{\prime} u_{i}^{\prime}\right\rangle / 2}{\partial t}= & -\left\langle w u_{i}^{\prime}\right\rangle \frac{\partial\left\langle u_{i}\right\rangle}{\partial z}-\left\langle w u^{\prime}\right\rangle \frac{\partial u_{s}}{\partial z}-\frac{g}{\langle\rho\rangle}\left\langle w \rho^{\prime}\right\rangle \\
& -\frac{\partial}{\partial z}\left\langle\frac{1}{2} u_{i}^{\prime} u_{i}^{\prime} w+\frac{1}{\langle\rho\rangle} w p^{\prime}\right\rangle+\langle\epsilon\rangle+\text { SGS }
\end{aligned}
$$

The left-hand side represents the temporal rate of change of TKE per unit mass (in the following discussion we will omit "per unit mass"). The rhs terms are from left to right 

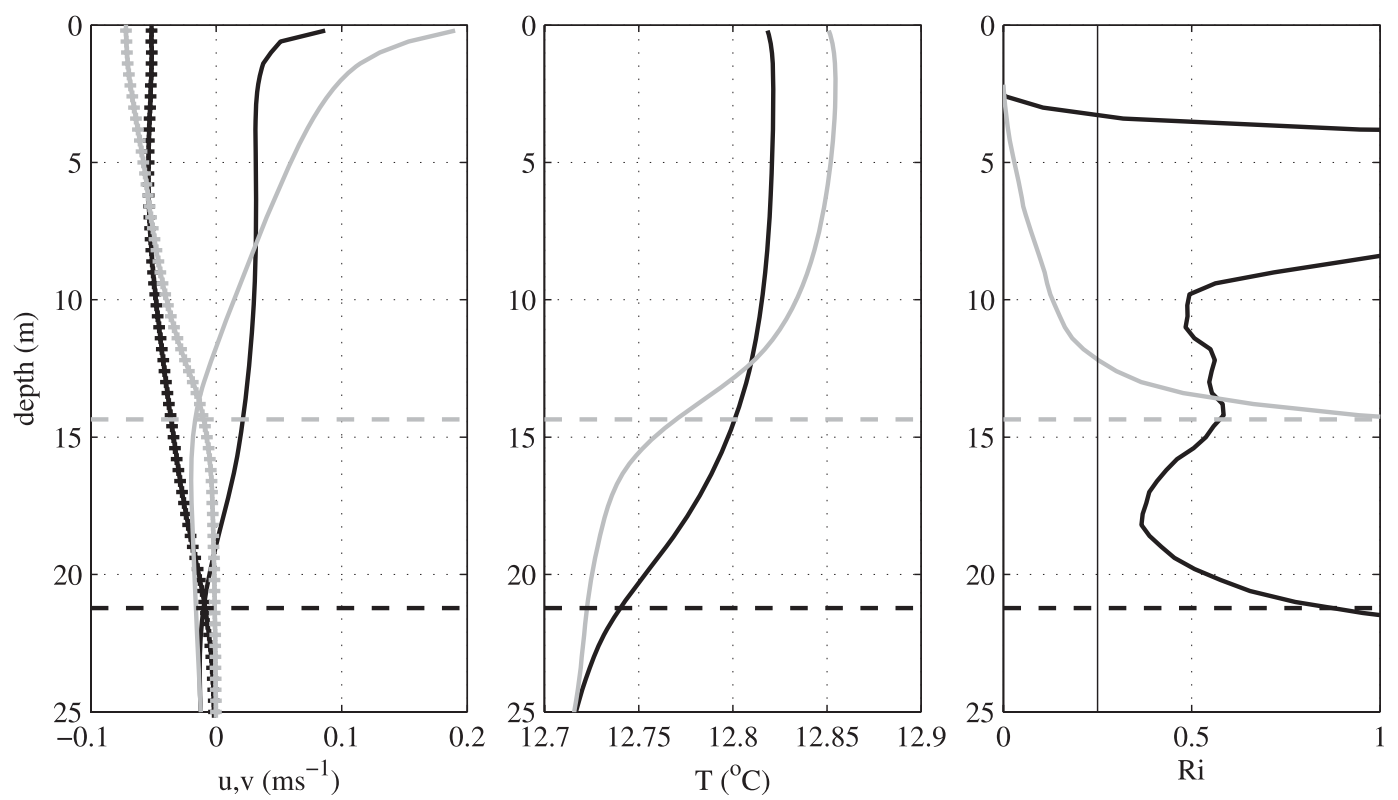

FIG. 5. Horizontally averaged LES solutions from the time of maximum mixed layer deepening (0800 PST, see Fig. 3) with (black) and without (gray) LC. (right) The Richardson number is calculated based on the (left) velocity $u$ (solid line) and $v$ (solid line with pluses) and (middle) temperature profiles according to (10); $\mathrm{Ri}=0.25$ (vertical thin black line) and $h$ (dashed lines).

TKE shear production rate converting Eulerian mean energy to TKE; TKE production rate by Stokes drift shear converting wave energy to TKE; buoyancy flux converting between potential energy and TKE; vertical divergence of energy fluxes due to vertical TKE advection (fourth term) and rate of turbulent pressure work (fifth term); $\epsilon$ the TKE dissipation rate; and SGS all remaining subgrid-scale terms (see, e.g., Skyllingstad et al. 2000). Details on the SGS model are described in appendix B. Note that, unlike in Reynolds-averaged Navier-Stokes equation models, the dominant flux and energy carrying turbulent eddies are resolved in the LES and do not need to be parameterized. The remaining SGS terms include the SGS shear production term $\left\langle\tau_{x z}^{\mathrm{SGS}}\right\rangle \partial\langle u\rangle / \partial z$, where the horizontally averaged SGS stress $\left\langle\tau_{x z}^{\mathrm{SGS}}\right\rangle$ is parameterized via the SGS closure scheme discussed in section $2 b$.

In this study we consider in detail only resolved terms in the TKE budget, except for the TKE dissipation rate. A budget residual is defined by subtracting all resolved terms from the TKE dissipation rate. This residual is typically about $11 \%$ of $\langle\epsilon\rangle$ below the first two near-surface grid points $(z<-0.6 \mathrm{~m})$ where SGS terms are less important. The magnitude of the residuals is consistent with previous LES experiments (Skyllingstad et al. 2000; Grant and Belcher 2009). Before examining TKE budgets for the whole 3-h wind event, we will first focus on TKE budgets for the time point from the previous section, when the rate of mixed layer deepening was greatest (at 0800 PST).

\section{1) TKE BUDGETS DURING RAPID MIXED LAYER DEEPENING}

Without LC, the dominant TKE balance is between shear production and dissipation (thick black dashed and solid lines, respectively, in Fig. 6), consistent with shear-driven boundary layer turbulence. As anticipated from the Ri profile, TKE shear production decreases from the surface to the base of the mixed layer. The remaining terms in (11) contribute generally little to the TKE balance. Although buoyancy fluxes (black line with asterisks) are small overall, this flux is critical in vertical temperature mixing and mixed layer deepening, as discussed in section 3a. Peak buoyancy fluxes are found in regions where temperature gradients are relatively large (so that temperature anomalies are relatively large) but not so large as to significantly dampen turbulence (so that vertical turbulent velocities can accomplish advective transport).

The TKE budget with LC differs significantly from the budget without LC (Fig. 7). Unlike shear-driven boundary layer turbulence, the dominant balance is generally not between shear production and dissipation because the Stokes drift shear contributes significantly to the TKE production (gray line with plus signs). Inspection of the TKE budget suggests three different turbulence regimes within the surface boundary layer: 1 ) a regime where both shear production and Stokes drift shear production are important in balancing dissipation that occurs in two 


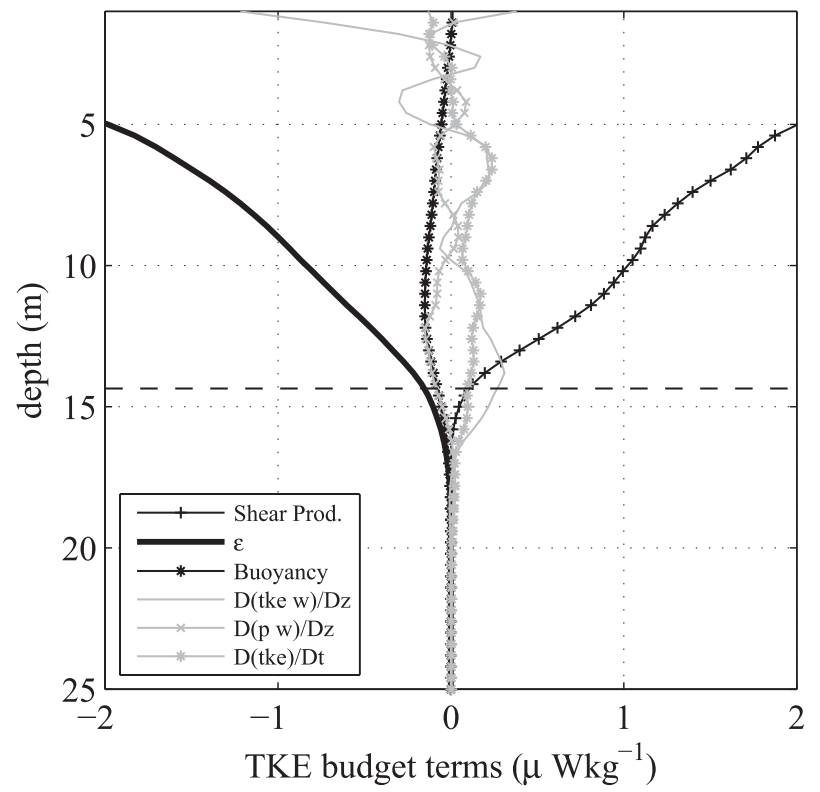

FIG. 6. Resolved terms of the TKE budget (11) without LC: TKE shear production (black line with pluses), $\langle\epsilon\rangle$ (thick solid black line), buoyancy flux (black line with asterisks), vertical divergence of TKE advection (gray line), vertical divergence of pressure work (gray line with crosses), and temporal rate of change of TKE (gray line with asterisks). Horizontal black dashed line indicates $h$.

regions very near the surface (to about 4-m depth) and near the middle of the mixed layer $(10-15 \mathrm{~m}) ; 2$ ) a regime where Stokes drift shear production is approximately balanced by dissipation, which occurs between $\sim 4$ and $10 \mathrm{~m}$ of depth (such a regime has been suggested previously; Polton and Belcher 2007; Grant and Belcher 2009); and 3) a thermocline erosion regime close to the base of the mixed layer, where shear production shows a local maximum and buoyancy fluxes are similar in magnitude to dissipation. The location of the peak in Eulerian TKE shear production closely coincides with the local maximum in velocity shear (cf. to left panel of Fig. 5); in this depth region Reynolds stresses are monotonically decreasing.

Langmuir circulation also plays a significant role in advectively redistributing TKE (thin gray solid line). Turbulent velocities advectively transport TKE from a highly energetic near-surface layer to less energetic locations below (note the negative to positive transition in the transport term near 3-m depth). Similarly, TKE is deposited at the mixed layer base from a more energetic layer above (negative to positive transition near 18-m depth).

\section{2) SCALing TKE BUdGets}

Many velocity scales have been introduced to characterize Langmuir turbulence based on observations,

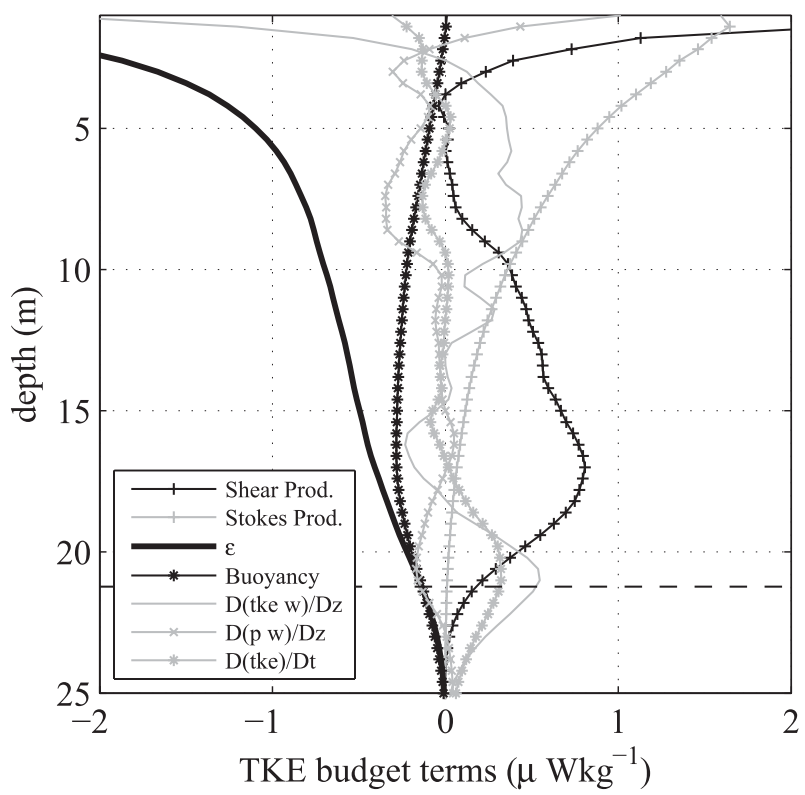

FIG. 7. Resolved terms of the TKE budget (11) with LC: TKE shear production (black line with pluses), TKE production by Stokes drift shear (gray line with pluses), $\langle\epsilon\rangle$ (thick solid black line), buoyancy flux (black line with asterisks), vertical divergence of TKE advection (gray line), vertical divergence of pressure work (gray line with crosses), and temporal rate of change of TKE (gray line with asterisks). Horizontal black dashed line indicates $h$.

theory, and numerical models (Harcourt and D'Asaro 2008; Grant and Belcher 2009; McWilliams and Sullivan 2000; Plueddemann et al. 1996; Smith 1996, 1998; Smyth et al. 2002). Building on these previous ideas, we will introduce a turbulent velocity scale $\mathcal{U}$ that is applicable to a wide range of wind and wave conditions in which turbulence can be predominantly driven by waves, Eulerian shear flow, or both processes. Grant and Belcher (2009) derive an expression for $\mathcal{U}$ for Stokes drift shear production dominated turbulence by equating an estimate for $\epsilon \approx \mathcal{U}^{3} / h$ with an estimate for the mean Stokes drift shear production

$$
h^{-1} \int_{-h}^{0}-\left\langle w u^{\prime}\right\rangle \frac{\partial u_{s}}{\partial z} d z \approx u_{*}^{2} u_{s 0} / h
$$

so that

$$
\mathcal{U}=\left(u_{*}^{2} u_{s 0}\right)^{1 / 3}=u_{*} \mathrm{La}_{t}^{-2 / 3},
$$

where $u_{s 0}=u_{s}(z=0 \mathrm{~m})$ is the surface Stokes drift, $\mathrm{La}_{t}=$ $\left(u_{*} / u_{s 0}\right)^{1 / 2}$ is the turbulent Langmuir number, and $h$ denotes the mixed layer depth. For this estimate of $\mathcal{U}$ it is assumed that turbulence does not extract energy from the Eulerian mean flow. Generally, however, turbulence, including wave-driven turbulence, converts mean 
Eulerian kinetic energy into TKE, as discussed in section $3 \mathrm{e}(3)$. To incorporate the TKE shear production into an estimate for $\mathcal{U}$, we approximate the mean TKE shear production as

$$
h^{-1} \int_{-h}^{0}-\left\langle w u^{\prime}\right\rangle \frac{\partial u}{\partial z} d z \approx u_{*}^{2} u_{0} / h
$$

where $u_{0}=u(z=0 \mathrm{~m})$ is the surface velocity. Equating now the total mean TKE production with $\epsilon$ yields

$$
\mathcal{U}=\left(u_{*}^{2} u_{s 0}+u_{*}^{2} u_{0}\right)^{1 / 3}=u_{*}\left(\mathrm{La}_{t}^{-2}+\frac{u_{0}}{u_{*}}\right)^{1 / 3}
$$

Note that for Stokes drift shear production-dominated turbulence, that is, $\mathrm{La}_{t} \rightarrow 0$, we recover the scaling (12) by Grant and Belcher (2009). For pure shear-driven turbulence, on the other hand, $\mathrm{La}_{t}^{-2}=0$, and we recover the velocity scaling of solid wall shear-driven boundary layer turbulence. An important implication of (13) is that in general $\mathcal{U}$ does not scale uniquely with $u_{*}$ and $\mathrm{La}_{t}$ alone, but also depends on $u_{0} / u_{*}$. This could in part explain observations by Smith (1999), which show that $\mathcal{U} / u_{*}$ scales with $u_{s 0} / u_{*}$ with an offset between different wind events (here $\mathcal{U}$ is the rms surface crosswind velocity). For each wind event $u_{0} / u_{*}$ could take a different value causing the observed offset.

Figure 8 indicates that terms of the TKE budget (11) scaled by $\mathcal{U}^{3} / h$ roughly collapse for different time points of the LC and no-LC runs. Each colored area encompasses one standard deviation centered around the 3-h time mean of the horizontally averaged TKE budgets. In spite of some variability over the full 3-h wind event (evidenced by the width of the shaded regions in Fig. 8), the main features discussed in section $3 \mathrm{c}(1)$ remain, such as a dominant balance between shear production and dissipation in the no-LC case and, in the LC case, a depth range with dominant Stokes drift shear production, as well as a local maximum in shear production near the base of the mixed layer (cf. also to Figs. 6, 7). In the remaining analysis, we will take advantage of the rich LES dataset to investigate more the resolved spatial structure of the turbulent fields and to elucidate mixing mechanisms.

\section{d. Spatially resolved turbulent structure and mixing mechanism}

Figure 9 shows horizontal cross sections of vertical velocities at $t=0800$ PST when the rate of mixed layer deepening is largest and at the $z$ location where the vertical velocity variance $\left\langle w^{2}\right\rangle$ is largest. In the simulations with Craik-Leibovich vortex force banded, coherent structures that are roughly aligned with the wind

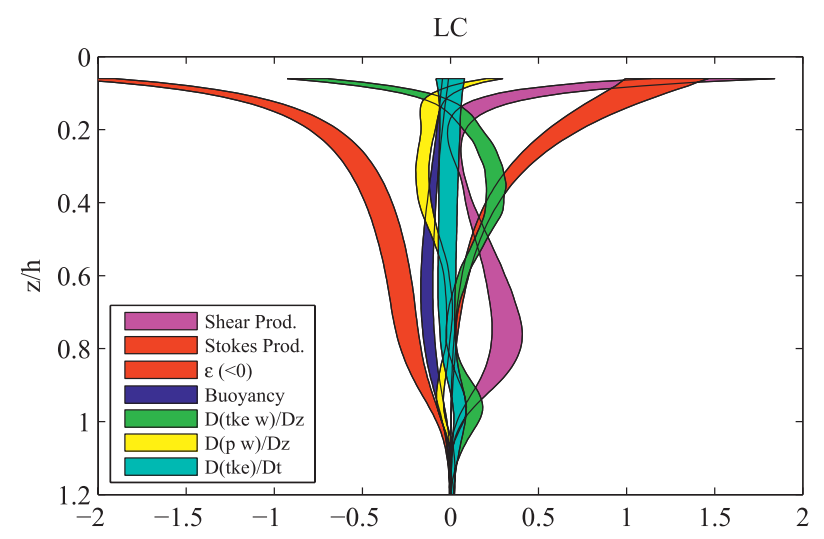

no LC

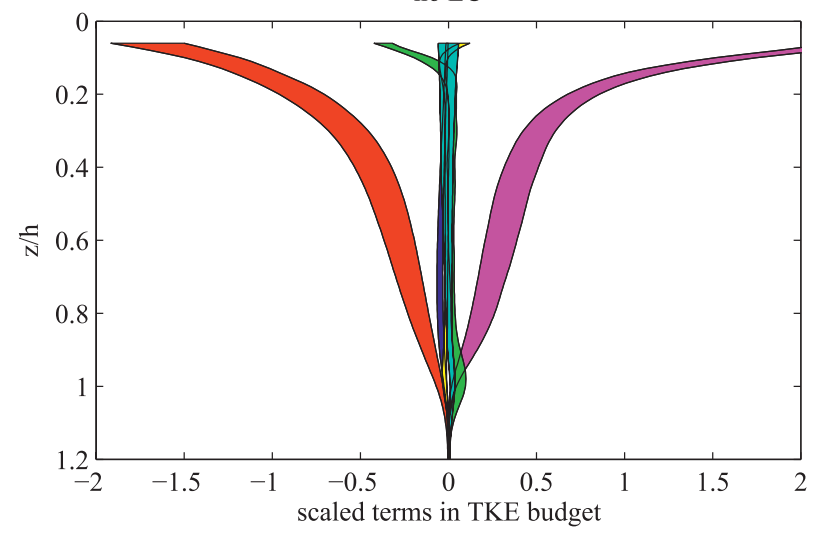

FIG. 8. Resolved terms of the TKE budget (11) scaled by $\mathcal{U}^{3} / h$ (top) with and (bottom) without LC: TKE shear production (magenta), TKE production by Stokes drift shear (red positive), $\langle\epsilon\rangle$ (red, negative), buoyancy flux (blue), vertical divergence of TKE advection (green), vertical divergence of pressure work (yellow), and temporal rate of change of TKE (cyan). Each colored area encompasses the standard deviation centered around the mean based on the profile time series.

are apparent (left panel). Rows of downwelling and upwelling velocities are due to LC roll vortices. The typical spacing between two adjacent LC cells is between 20 and $40 \mathrm{~m}$, consistent with $l_{\mathrm{LC}}=34 \mathrm{~m}$ from Fig. 3, although the individual cell spacing is irregular and variable. The LC simulation captures the LC characteristic of narrower and faster downwelling regions (see, e.g., Leibovich and Paolucci 1981). Downward vertical velocities with magnitude greater than $2\left\langle w^{2}\right\rangle^{1 / 2}$ are commonly found in downwelling regions of strong LC cells. This LC feature will be used in section 3e to identify LC. Without LC, downwelling jets are more isolated, localized, and randomly distributed structures (right panel of Fig. 9). The presence of relatively small ( $<10 \mathrm{~m})$ vertical velocity dipoles indicates that the distance to the surface (about $5 \mathrm{~m}$ ) limits the eddy size in shear-driven boundary layer turbulence. Different eddy sizes in the 


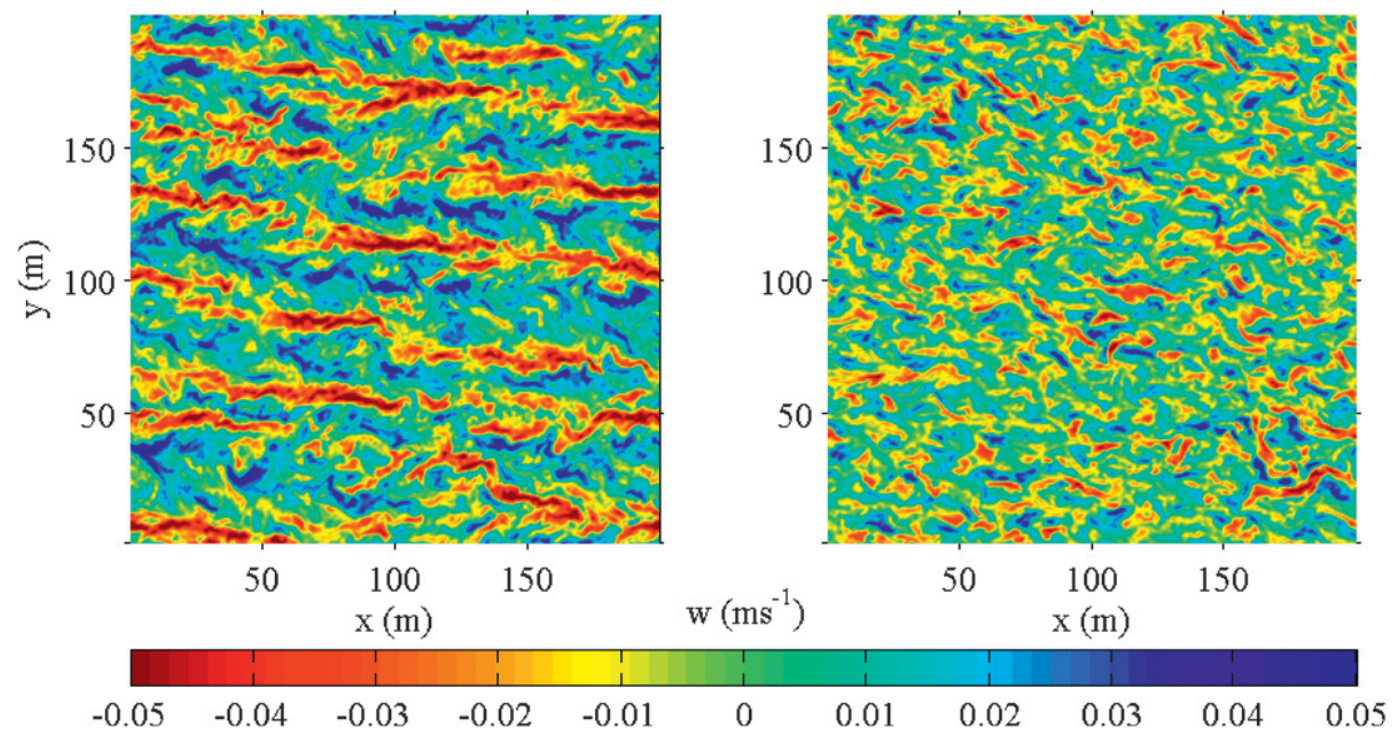

FIG. 9. Vertical velocities at 0800 PST (left) with and (right) without LC at depth $z=6.2$ and $4.6 \mathrm{~m}$, respectively, where the vertical velocity variance has a maximum values with $\left\langle w^{2}\right\rangle^{1 / 2}=0.021$ and $0.015 \mathrm{~m} \mathrm{~s}^{-1}$, respectively. A threshold of $w<-2\left\langle w^{2}\right\rangle^{1 / 2} \approx 0.04 \mathrm{~m} \mathrm{~s}^{-1}$ (dark red in left panel) indicates strong LC activity.

LC and no-LC cases and the presence of coherent LC structures significantly influences the temperature distribution.

Temperature $x-z$ cross sections (Fig. 10, again at 0800 PST) visually emphasize turbulent processes near the thermocline, which control mixed layer deepening. Strong temperature gradients at the thermocline roughly coincide with the isothermal surface at $T \approx 12.78^{\circ} \mathrm{C}$ (yellow), which we will refer to as "thermocline surface." In the noLC case, Kelvin-Helmholtz (KH) shear instabilities are confined to the vicinity of the thermocline and billows break "locally" (bottom panel). Note that we refer to the generalized form of $\mathrm{KH}$ instabilities, which is concerned with "the interplay of the stabilizing influence of gravity on a continuously stratified fluid and of the destabilizing influence of basic shear" (Drazin and Reid 2004), simply as KH instability. With LC, the thermocline surface is more strongly displaced, often extending well above the mean mixed layer depth $(h \approx 21 \mathrm{~m})$. Our interpretation is that cool cusps of breaking KH waves are advected upward by LC into the mixed layer where they disperse laterally and contribute to mixing. These processes could be responsible for the observed density blobs (Fig. 4).

It is insightful to consider now the full three-dimensional structure of the flow fields (Figs. 11, 12). The deepest LC upwelling regions are located in between strong downwelling regions and are indicated by along-wind rows of relatively cool temperatures near the thermocline at $z=$ $h=21 \mathrm{~m}$ (Fig. 11). Cusps of breaking KH waves (visible in the $x-z$ cross section) are "sucked" upward into LC upwelling region as cooler water in the $y-z$ cross section appears in these upwelling regions. Thus, Langmuir turbulence has a critically three-dimensional structure with coherent cell structure in the crosswind depth plane and $\mathrm{KH}$ instabilities in the along-wind depth plane. This stands in great contrast to the no-LC case in which $\mathrm{KH}$ instabilities are the dominant feature without alternating upwelling and downwelling regions in the crosswind direction (Fig. 12).

These spatial temperature and vertical velocity snapshots together with the previous analyses of horizontally averaged temperature, velocity, and TKE budgets suggest that the combination of LC and $\mathrm{KH}$ instabilities provide an efficient mechanism to deepen the mixed
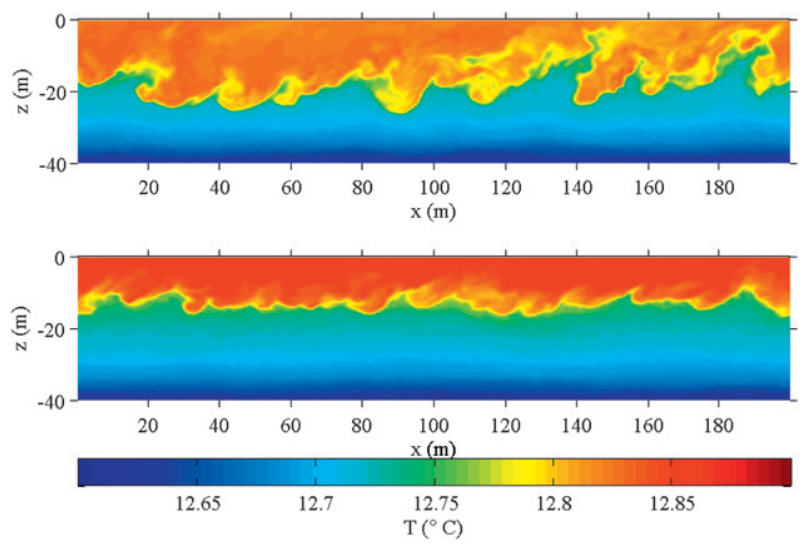

FIG. 10. Depth vs along-wind temperature cross sections (top) with and (bottom) without LC at 0800 PST. 

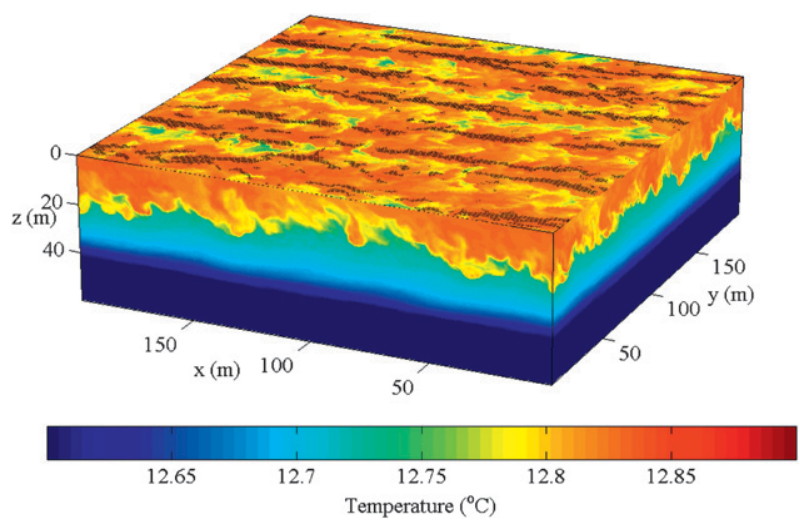

FIG. 11. Volume plot of simulated temperature with LC at 0800 PST. The horizontal plane shows the temperature cross section at $z=h$ and has been for improved visualization plotted at $z=0$. Black dots correspond to horizontal locations of LC downwelling regions with $w<-\left\langle w^{2}\right\rangle^{1 / 2}$ at the depth where vertical velocity variance is a maximum.

layer, which can be described as a three step process. In the first step, momentum is efficiently transported downward by LC to the base of the mixed layer. In the next step, enhanced shear causes elevated $\mathrm{KH}$ instabilities at the base of the mixed layer, eroding the thermocline. In the final step, colder eroded thermocline water is transported upward and mixed into the nearsurface layer by LC. The key aspect of this mechanism is that mixing processes due to $\mathrm{LC}$ and $\mathrm{KH}$ act together. In the following subsection we will investigate this mechanism further.

\section{e. Conditionally averaged LC cells}

Since snapshots of turbulent fields are irregular and variable in space and time (Figs. 9, 10), we will introduce conditional averages, similar to the work by McWilliams et al. (1997). However, since our fields are nonstationary and we simulated only one realization of the wind event, unlike McWilliams et al. (1997), we need to extract statistics from a spatial snapshot. To do this, we take advantage of the fact that the LC is roughly aligned with the wind (Fig. 9) so that conditional averages can be defined as function of depth and crosswind direction. To average over many LC events, it is necessary to specify a common feature of the LC that can be identified in the simulated dataset. Figure 9 indicates that downwelling jets with $w<-2\left\langle w^{2}\right\rangle^{1 / 2}$ at the depth where vertical velocity variance is a maximum robustly characterize the presence of LC. Therefore, we use this vertical velocity threshold to specify horizontal locations $\left(x^{i}, y^{i}\right)$ of LC features. The conditional average $\{\cdots\}$ of any quantity $Q(x, y, z, t)$ can be expressed as

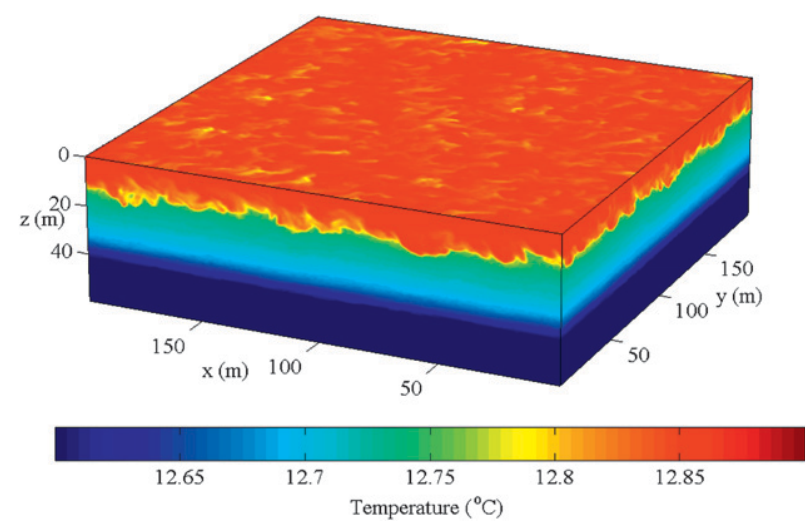

FIG. 12. Volume plot of simulated temperature without LC at 0800 PST. The horizontal plane shows the temperature cross section at $z=h$ and has been for improved visualization plotted at $z=0$.

$$
\{Q\}(y, z, t)=\frac{1}{n} \sum_{i=1}^{n} Q\left(x^{i}, y-y^{i}, z, t\right),
$$

where $n$ is the number of LC feature (downwelling) locations.

The width of the conditionally averaged LC cell, $l_{c}$, is determined such that there is no net flow through horizontal planes and no horizontal velocity in upwelling centers; that is,

$$
\begin{gathered}
\int_{-l_{c} / 2}^{l_{c} / 2}\{w\}(y, z) d y \approx 0 \quad \text { and } \\
\{v\}\left(y= \pm l_{c} / 2, z\right) \approx 0 .
\end{gathered}
$$

From about 0730 to 0810 PST, when the mixed layer deepening is largest, $l_{c}$ is approximately proportional to the dominant LC length scale $l_{\mathrm{LC}}$ based on spectral velocity estimates (Fig. 3). Note that for some time periods $l_{c}$ varies rapidly and assumes relatively large values. This is because conditionally averaged upwelling velocities are relatively weak in the presence of multiple LC cell sizes. In these cases, $l_{c}$ based on (15) does not provide a robust estimate of the average LC width.

Based on the conditional averages, we may decompose the resolved turbulent velocities $u_{i}^{\prime}$ into one part due to coherent LC motion as extracted by conditional averaging and one part due to remaining fluctuations $u_{i}^{\prime \prime}$,

$$
u_{i}^{\prime} \equiv\left\{u_{i}\right\}^{\prime}+u_{i}^{\prime \prime}
$$

Note that $u_{i}^{\prime \prime}$ includes incoherent turbulence and all coherent motions not captured in $\left\{u_{i}\right\}^{\prime}$, such as smaller-scale 
LC. Therefore, the analysis of conditionally averaged LC cells focuses only on the LC contributions at the dominant scale, although observations and simulations indicate the presence of a hierarchy of cell sizes (Smith 1992; Plueddemann et al. 1996; Kukulka et al. 2009). Note also that the decomposition (16) based on the conditional average (14) is generally not physically meaningful because $\left\{u_{i}\right\}^{\prime}$ is defined for a single LC cell, whereas $u_{i}^{\prime}$ is defined for the whole domain. However, dominant LC cells around 0800 PST approximately fill the whole domain with a spacing of roughly $l_{c}=34 \mathrm{~m}$ (see also Figs. 3, 9), so it is reasonable to assume that the flow in the volume encompassing the conditionally averaged LC cell has the same statistical properties as the flow in the whole domain. The following discussion focuses on the flow field of a single conditionally averaged LC cell from $y=-l_{c} / 2$ to $y=l_{c} / 2$ at 0800 PST.

\section{1) TEMPERATURE AND VELOCITIES}

The conditionally averaged velocity field projected into the depth-crosswind plane (Fig. 13) resembles the idealized flow pattern of two-dimensional LC with faster, narrower downwelling and slower, broader upwelling regions (e.g., Leibovich and Paolucci 1981). Unlike unstratified conditions, for which downwelling jets are controlled by an Ekman layer depth scale (Polton and Belcher 2007), the vertical extent of LC downwelling jets is confined by the thermocline in this study. Relatively warm surface water is advected into downwelling regions, while cooler near-thermocline water is transported upward. The correlation of temperature anomaly $\{T\}^{\prime}=\{T\}-\langle\{T\}\rangle$ and vertical velocity suggests a significant vertical temperature transport $\left\langle\{T\}^{\prime}\{w\}\right\rangle$ associated with dominant scale LC motion (bottom panel, Fig. 13). Indeed, the transport is controlled by the dominant scale LC ( $>50 \%)$ down to a depth of about $11 \mathrm{~m}$ (Fig. 14), that is, in the depth region where temperature profiles are nearly homogeneous (see for comparison Fig. 5) and TKE shear production is weak (Fig. 7). Near the base of the mixed layer, the overall contribution of dominant scale LC temperature transport is relatively small because $\mathrm{KH}$ instabilities erode and mix the thermocline there.

Are dominant scale LC currents energetic enough to engulf denser thermocline water against gravity up into the mixed layer? The kinetic energy of a vertically traveling water parcel is approximately converted to potential energy after a travel distance $L \approx W / N$, where $N$ is the buoyancy frequency and $W$ is a vertical LC velocity scale. Estimating the temperature gradient at the thermocline as $0.05^{\circ} \mathrm{C}(5 \mathrm{~m})^{-1}$ and $W \approx 5 \times 10^{-3} \mathrm{~m} \mathrm{~s}^{-1}$ results in $L \approx 1 \mathrm{~m}$, which is significantly smaller than the
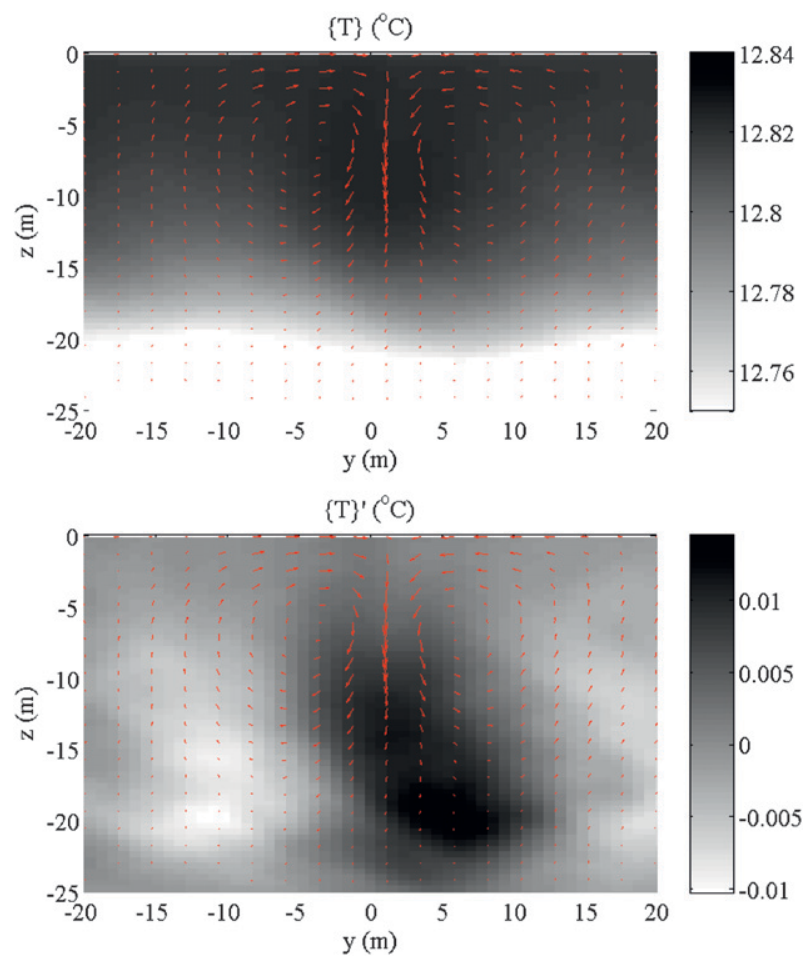

FIG. 13. (top) Conditionally averaged temperatures $\{T\}$ and (bottom) its anomaly $\{T\}^{\prime}=\{T\}-\langle\{T\}\rangle$. Arrows show velocities projected in to the $y-z$ plane. The longest velocity arrow indicates a speed of $0.05 \mathrm{~m} \mathrm{~s}^{-1}$.

vertical extent of the thermocline ( $>5 \mathrm{~m}$, see Fig. 5). This suggests that an engulfment process by dominant scale LC alone is not effective in eroding the thermocline.

A qualitatively similar, but more pronounced, pattern arises for the along-wind velocity. An along-wind velocity jet in the center of the LC convergence region extends vertically down to the base of the mixed layer (Fig. 15). Anomalies $\{u\}^{\prime}$ (bottom panel) correlate well with vertical velocities $\{w\}$. This results in a significant momentum transport $\left\langle\{u\}^{\prime}\{w\}\right\rangle$ by dominant-scale LC in depth ranges between 4 and $12 \mathrm{~m}$ (right panel in Fig. 14), where $\langle u\rangle$ is nearly constant (for comparison, see Fig. 5). The along-wind velocity jet in the downwelling region leads to locally enhanced vertical shear at the base of the mixed layer. That the LC contributes significantly to the formation of enhanced vertical shear and the along-wind velocity jets in the mixed layer is consistent with the observations from Weller and Price (1988). Enhanced shear below LC convergence regions, in our study found near the base of the mixed layer, are also present in shallow water Langmuir "supercells" near the ocean bottom (Gargett et al. 2004), providing a mechanism for sediment resuspension. We will next investigate if this shear can overcome stabilizing stratification to facilitate the generation of $\mathrm{KH}$ instabilities. 

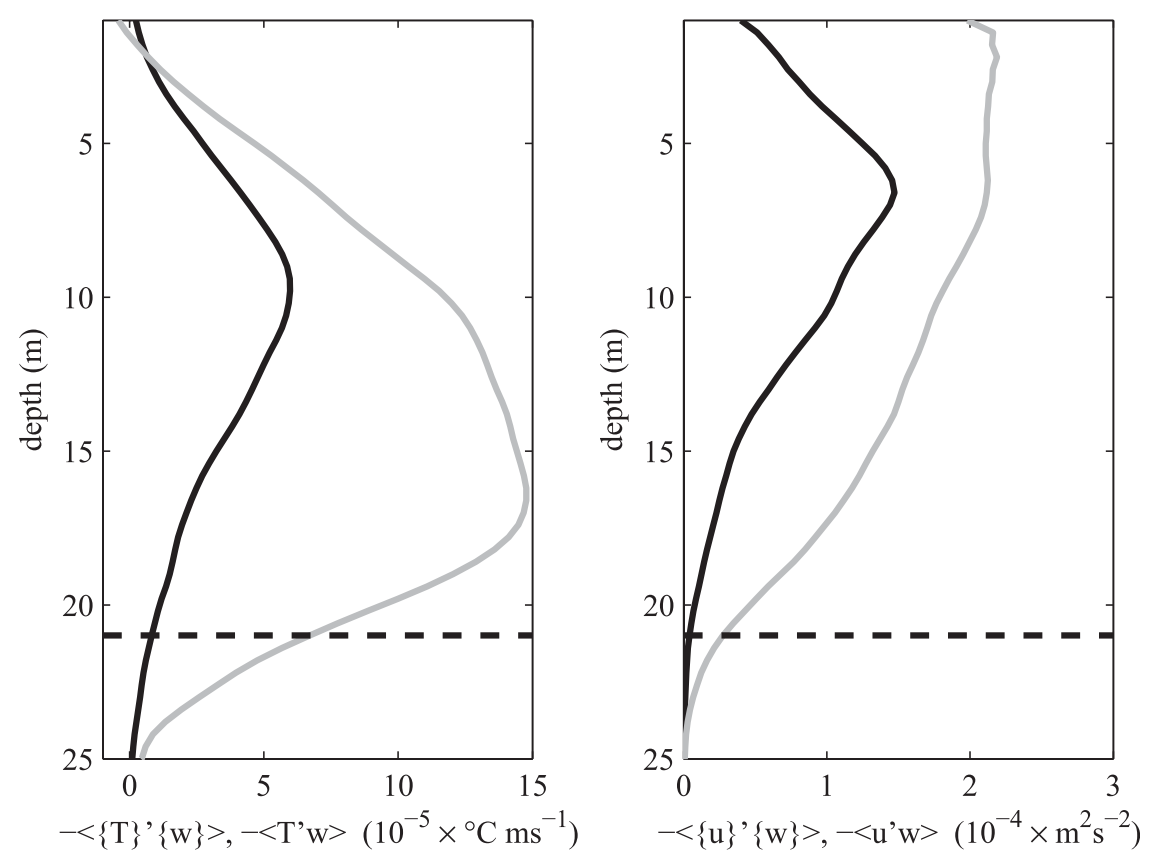

FIG. 14. Vertical advective turbulent transport for LC solutions for (left) temperature and (right) along-wind momentum: transport by the dominant scale LC (black lines), total turbulent transport (gray lines), and $h$ (dashed lines).

\section{2) ENHANCED SHEAR INSTABILITES BELOW LC DOWNWELLING JETS}

To examine the competition between vertical shear of horizontal mean flow and stratification, we will introduce a Richardson number $\{\mathrm{Ri}\}$ analogous to (10), but with horizontal averages of density and velocity replaced by conditional averages. The Richardson number $\{\mathrm{Ri}\}$ of the conditionally averaged fields indeed has a local minimum with $\mathrm{Ri}<0.25$ below the downwelling region close to the base of the mixed layer, indicating that shear instabilities are enhanced there (top panel of Fig. 16). The depth range of relatively low $\{\mathrm{Ri}\}$ is consistent with small Ri based on horizontal averages (cf. right panel of Fig. 5). Note that the locally enhanced vertical shear of horizontal velocities below downwelling regions is isolated in the conditional averages but "smeared" in the horizontal averages so that the horizontally averaged Ri remains above the critical value (see Fig. 5). Conditionally sampled dissipation rates $\{\epsilon\}$ averaged over a depth range with $\mathrm{Ri}<0.5$ near the thermocline (between the two dashed lines in top panel of Fig. 16) indicate enhanced turbulence levels close to the horizontal velocity jet (bottom panel of Fig. 16). Note, however, that much detail of the actual spatial distribution of $\epsilon$ is lost through the conditional averaging procedure (Fig. 17). Instantaneous TKE dissipation rates are highly variable, differing by several orders of magnitude at a given depth. Interestingly, $\epsilon$ can be clearly enhanced below LC downwelling regions near the thermocline (sharp temperature gradients are roughly where the color transitions from blue to green at $z \approx 21 \mathrm{~m}$ ), indicating that elevated turbulence levels there are not simply due to downward advection of enhanced surface turbulence but, rather, generated locally. Therefore, locally enhanced shear instabilities are likely responsible for the previously discussed augmented shear production at the base of the mixed layer (Fig. 7).

Enhanced shear instabilities beneath downwelling regions of the LC as a mechanism for thermocline erosion have been suggested previously by Li and Garrett (1997), but to our knowledge these are the first results providing concrete evidence for this idea. Analysis of conditionally averaged fields confirms the mixed layer deepening mechanism proposed in section $3 \mathrm{~d}$ : LC transports momentum efficiently downward so that the LC preconditions enhanced shear instabilities at the base of the mixed layer. In turn, $\mathrm{KH}$ instabilities erode the thermocline and precondition the temperature field for enhanced advective LC transport. Such coupled dynamics of LC and $\mathrm{KH}$ instabilities are responsible for the observed enhanced mixed layer deepening. Since LC is one critical component in driving mixed layer deepening, it is interesting to investigate LC energetics more closely. 
$\{\mathrm{u}\}(\mathrm{m} / \mathrm{s})$

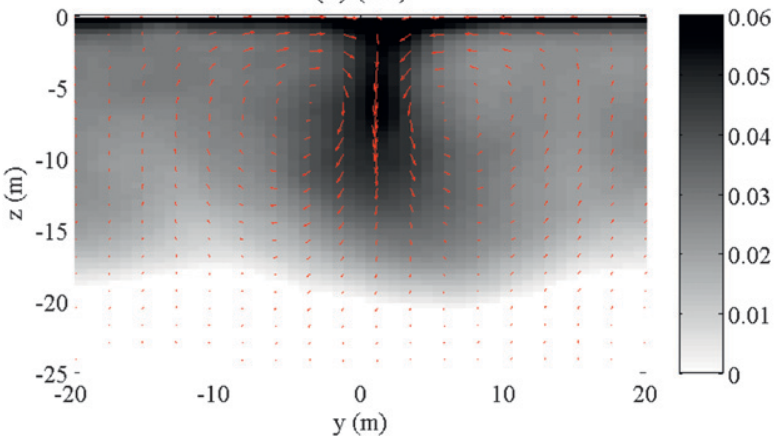

$\{\mathrm{u}\}^{\prime}(\mathrm{m} / \mathrm{s})$

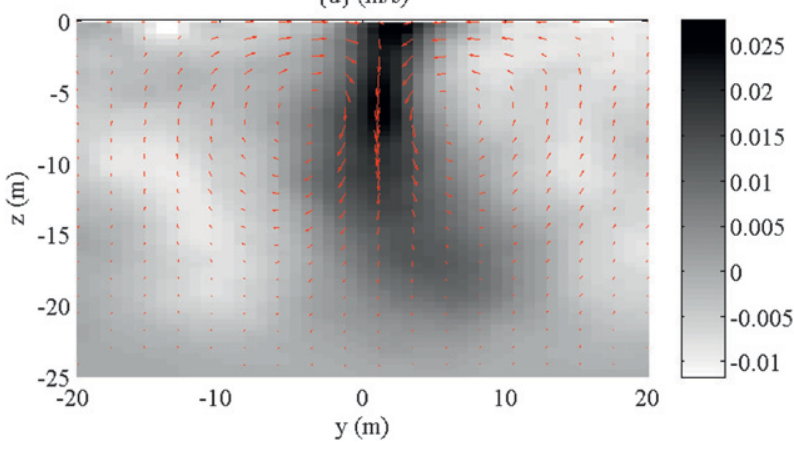

FIG. 15. (top) Conditionally averaged along-wind velocity $\{u\}$ and (bottom) its anomaly $\{u\}^{\prime}=\{u\}-\langle\{u\}\rangle$. Arrows show velocities projected in to the $y-z$ plane. The longest velocity arrow indicates a speed of $0.05 \mathrm{~m} \mathrm{~s}^{-1}$.

\section{3) ENERGETICS OF THE DOMINANT LC SCALE}

Based on the decomposition (16) one may derive an energy budget of the dominant scale LC motion $\left\{u_{i}\right\}^{\prime}$ with the two production terms,

$$
-\left\langle\{w\}^{\prime}\left\{u_{i}\right\}^{\prime}\right\rangle \frac{\partial\left\langle u_{i}\right\rangle}{\partial z} ; \quad-\left\langle\{w\}^{\prime}\{u\}^{\prime}\right\rangle \frac{\partial u_{s}}{\partial z},
$$

due to Eulerian mean shear and Stokes drift shear, respectively. Here repeated indices are summed over, $v_{s}=0$ and $\langle\{X\}\rangle \approx\langle X\rangle$. Note that the full TKE budget of the dominant scale LC motion also includes nonlinear terms
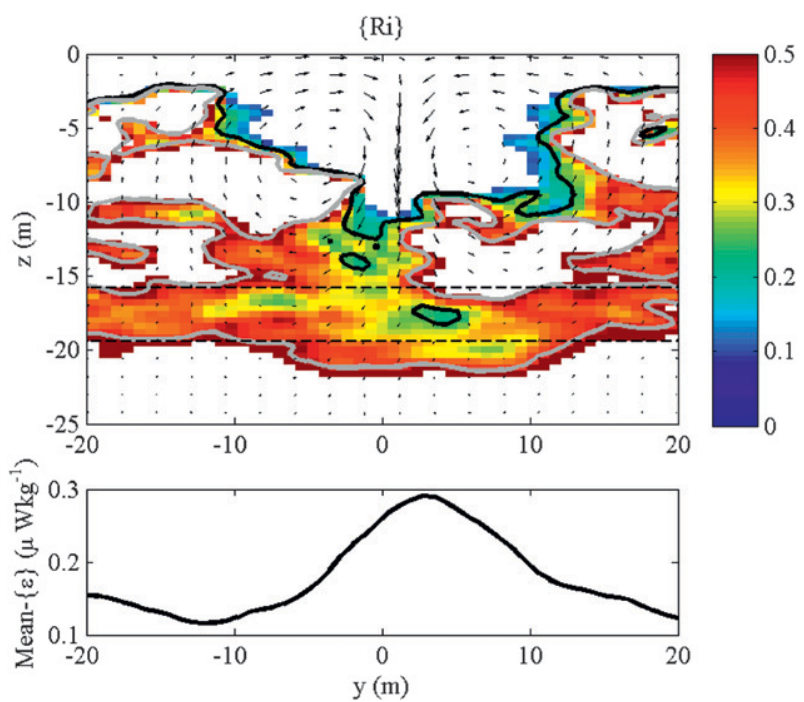

FIG. 16. (top) Conditionally averaged Richardson number $\{\mathrm{Ri}\}$. Thick solid lines show contours of $\{\mathrm{Ri}\}=0.25$ (black) and $\{\mathrm{Ri}\}=0.50$ (gray). Values with $\{\mathrm{Ri}\}>0.6$ and $\{\mathrm{Ri}\}<0.1$ are white. Arrows show velocities $(v, w)$ projected onto the $y-z$ plane. The longest velocity arrow indicates a speed of $0.05 \mathrm{~m} \mathrm{~s}^{-1}$. Dashed horizontal lines at $z=-15.8 \mathrm{~m}$ and $z=-19.4 \mathrm{~m}$ show $\mathrm{Ri}<0.5$ based on Fig. 5 . (bottom) Mean conditionally averaged TKE dissipation rate $\{\epsilon\}$ between depths 15.8 and $19.4 \mathrm{~m}$. Since $\epsilon$ varies by several orders of magnitude, averages have been obtained in log space.

that transfer energy between scales associated with the flow fields $\left\{u_{i}\right\}^{\prime}$ and $u_{i}^{\prime \prime}$. Both production terms can be compared to the corresponding production terms in the total TKE budget (11). Down to a depth of about $12 \mathrm{~m}$, the dominant scale LC motion contributes roughly $20 \%$ to the total TKE with a peak contribution of $33 \%$ at $z=$ $-6 \mathrm{~m}$ (Fig. 18). Although not the dominant source of TKE, these coherent motions still dominate the advective vertical transport (see previous subsection) because their relatively large size makes them efficient mixers.

The dominant scale of LC motion extracts less energy from the mean motion and wave motion than the remaining motion $u_{i}^{\prime \prime}$ (a combination of smaller scale coherent and incoherent turbulence) does:

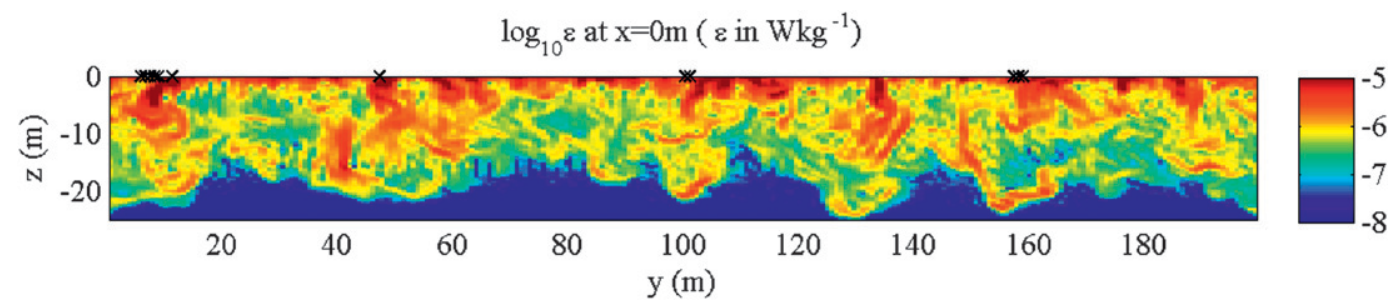

FIG. 17. Instantaneous depth vs crosswind cross section of dissipation rate $\epsilon$ at $x=0 \mathrm{~m}$. Crosses at the air-sea interface indicate LC downwelling locations $y^{i}$ below which one can typically observe enhanced dissipation rates near the thermocline at $z \approx 21 \mathrm{~m}$. 

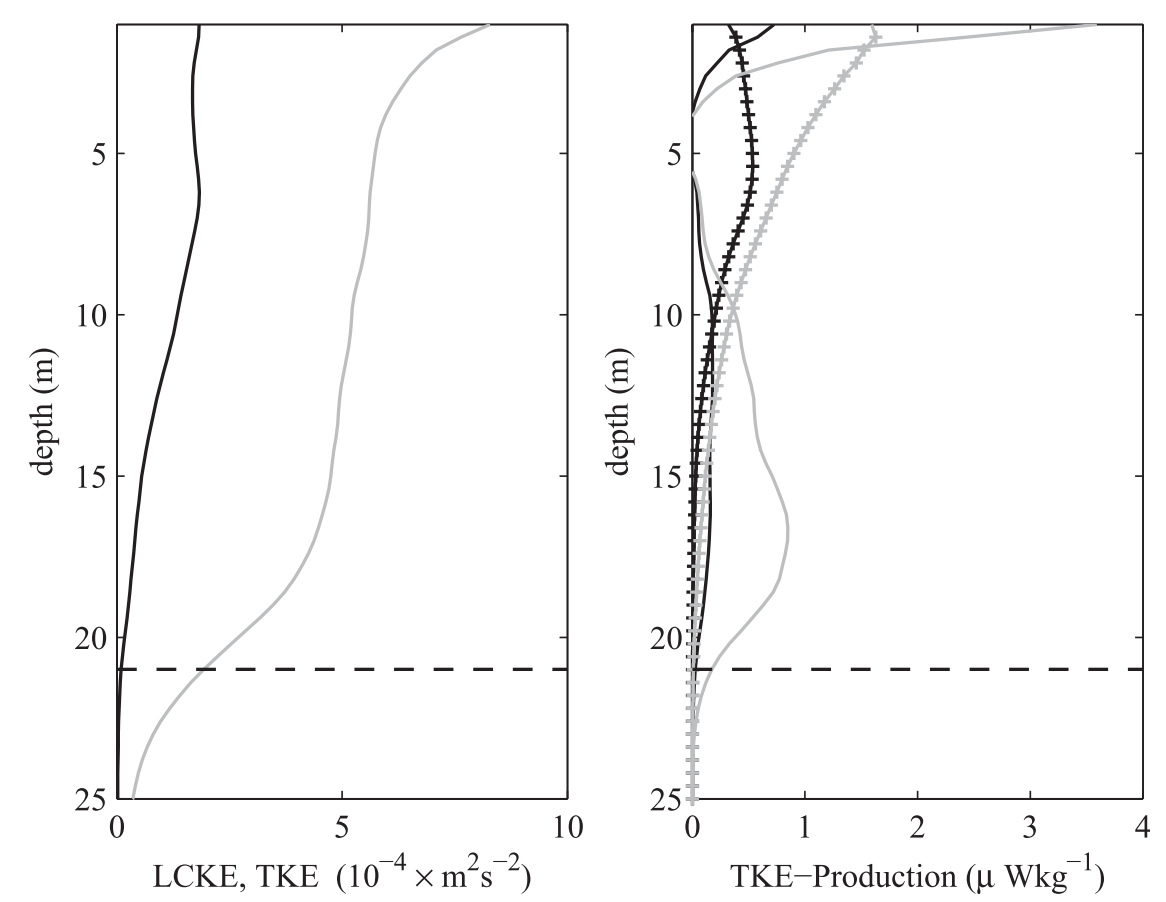

FIG. 18. (left) Total turbulent kinetic energy (gray line) and (right) its production (gray lines) according to (11) compared to (left) $1 / 2\left\langle\left\{u_{i}\right\}^{\prime}\left\{u_{i}\right\}^{\prime}\right\rangle$ [dominant scale LC kinetic energy (LCKE) (black line)] and (right) its associated production terms (black lines); production term by mean Eulerian shear (lines without symbols) and by Stokes drift shear (lines with pluses); and $h$ (dashed horizontal lines).

dominant scale LC shear production total shear production

$$
=\frac{\int_{-H}^{0}-\left\langle\{w\}^{\prime}\left\{u_{i}\right\}^{\prime}\right\rangle \frac{\partial\left\langle u_{i}\right\rangle}{\partial z} d z}{\int_{-H}^{0}-\left\langle w^{\prime} u_{i}^{\prime}\right\rangle \frac{\partial\left\langle u_{i}\right\rangle}{\partial z} d z}=22 \%
$$

and

dominant scale LC Stokes production

$$
\begin{gathered}
\text { total Stokes production } \\
=\frac{\int_{-H}^{0}-\left\langle\{w\}^{\prime}\{u\}^{\prime}\right\rangle \frac{\partial u_{s}}{\partial z} d z}{\int_{-H}^{0}-\left\langle w^{\prime} u^{\prime}\right\rangle \frac{\partial u_{s}}{\partial z} d z}=43 \% .
\end{gathered}
$$

Here $H$ indicates the total ocean depth. It is not surprising that the dominant scale LC motion extracts a relatively small fraction of the total turbulent shear production because the mean Eulerian shear is strongest near the surface and near the mixed layer where $\{w\}^{\prime}$ is small. That the dominant scale LC Stokes production is only $43 \%$ of the total Stokes production reflects that smaller scale turbulence (both coherent and incoherent) converts wave energy into turbulence close to the surface where the Stokes drift gradient is largest. Comparing the production profiles for the dominant scale LC motion (Fig. 18) indicates that the Eulerian mean shear flow also significantly drives dominant-scale LC motion; in fact,

dominant scale LC shear production

$$
\begin{gathered}
\overline{\text { dominant scale LC Stokes production }} \\
=\frac{\int_{-H}^{0}-\left\langle\{w\}^{\prime}\left\{u_{i}\right\}^{\prime}\right\rangle \frac{\partial\left\langle u_{i}\right\rangle}{\partial z} d z}{\int_{-H}^{0}-\left\langle\{w\}^{\prime}\{u\}^{\prime}\right\rangle \frac{\partial\left\langle u_{S}\right\rangle}{\partial z} d z}=85 \%
\end{gathered}
$$

This result confirms the significance of our velocity scaling (13), which incorporates production by Eulerian mean shear and Stokes drift shear.

\section{Summary and conclusions}

We have performed large-eddy simulations of an observed wind event during which the mixed layer significantly deepened and Langmuir circulations developed. The simulations have been validated based on 
data from a previous field experiment, SWAPP. The simulated mixed layer deepening and crosswind velocity variations are only consistent with observations if the Craik-Leibovich vortex force is included in the model. The Craik-Leibovich vortex force leads to the generation of Langmuir circulations through the interaction of sheared mean currents with surface gravity waves. Therefore, our results indicate that the Langmuir circulation plays a significant role in upper-ocean turbulence dynamics.

From the analysis of (i) instantaneous spatial fields, (ii) horizontally averaged profiles, (iii) kinetic energy balances, and (iv) conditional averages that depict a typical LC flow structure, we have identified fundamental differences in mixing between purely sheardriven turbulence and turbulence with LC. In the former case, turbulent kinetic energy is produced by KelvinHelmholtz shear instabilities and is largest near the surface, gradually decreasing to zero near the base of the mixed layer. This stands in contrast to the LC case in which at middepth range TKE production can be dominated by Stokes drift shear. Furthermore, the Eulerian mean shear peaks near the base of the mixed layer, so TKE production by mean shear flow is elevated there. The LC transports horizontal momentum efficiently downward, leading to a localized along-wind velocity jet below LC downwelling regions at the base of the mixed layer. This jet strongly enhances KH instabilities locally, which efficiently erodes the thermocline. In turn, enhanced breaking internal $\mathrm{KH}$ waves inject cold temperature perturbations into the mixed layer where LC currents are stronger, leading to enhanced advective temperature transport by the LC. Thus, the LC and KH instabilities work intimately together to strongly facilitate the mixed layer deepening process.

For a simple conceptual picture of this coupling, it is insightful to imagine each mixing process in isolation. Assume as initial condition a well-established mixed layer with a temperature and velocity jump at the thermocline (Fig. 19). By themselves KH instabilities would smooth the sharp gradients, but mixing would remain a local process confined to the thermocline and thermocline water would be only mixed slowly into the upper mixed layer. Next, consider LC alone without the presence of $\mathrm{KH}$ instabilities at the base of the mixed layer. In this case, the LC would be largely confined to the mixed layer because the relatively weak deeper LC current cannot overcome buoyancy forces at the thermocline. Therefore, LC currents would mostly transport already mixed water within the mixed layer. Finally, consider both mixing processes acting together: LCs transport momentum downward so that enhanced $\mathrm{KH}$ instabilities can erode the thermocline. Eroded thermocline water is then

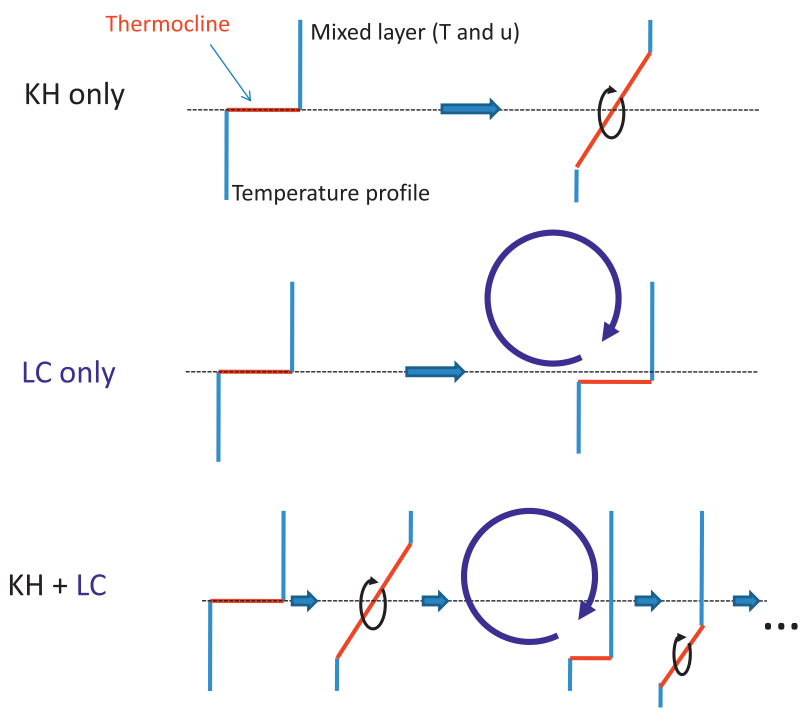

FIG. 19. Schematic of the tight coupling of two mixing processes.

transported upward by the LC so as to maintain the mixed layer.

LCs and the mechanism described in this study could provide a physical foundation for 1D ocean column models with a slab mixed layer (e.g., Price et al. 1986). These models prescribe a completely homogenized mixed layer with shear concentrated at the mixed layer base and deepening controlled primarily by shear instability. If LCs are critical to mixed layer homogenization and deepening, then 1D mixed layer models generally should depend on surface gravity wave effects. An open research question remains how mixed layer models can be improved by including surface wave properties explicitly (see, e.g., discussion by Li and Garrett 1997).

Clearly, more research is necessary to understand the mixed layer deepening processes for a variety of wind and wave conditions. In particular, it will be interesting to examine conditions under which breaking waves, which likely played a secondary role in this study, need to be taken into account. Breaking waves may be critical in the dynamics of shallower mixed layers and in strongly forced wind (e.g., hurricane) conditions. The study by Sullivan et al. (2007) alludes to the intricate interplay between Langmuir circulations and breaking waves, which could disperse (by injecting TKE) or catalyze LC (by seeding vertical vorticity) Langmuir circulations. Furthermore, LCs might significantly redistribute enhanced TKE in the near-surface layer. We will investigate these breaking wave effects with its implications on mixed layer dynamics in future studies.

Acknowledgments. This research was supported by the Office of Naval Research through Grants 
N00014-09-M-0112 (TK) and N00014-06-1-0178 (AP, JT). Author TK also received support from a Woods Hole Oceanographic Institution Cooperative Institute for Climate and Ocean Research Postdoctoral Scholarship. Computing resources were provided from the National Center for Atmospheric Research, which is sponsored by the U.S. National Science Foundation. We thank Gene Terray, Tom Farrar, and Jerry Smith for stimulating discussions. Two anonymous reviewers provided constructive comments.

\section{APPENDIX A}

\section{Vertically Integrated Transport}

To better understand the dynamics of the complex LES solutions, we will focus here on the role of the CraikLeibovich force and time-varying wind stress in the vertically integrated transport, which can be expressed in a closed-form analytic solution (see also Gnanadesikan and Weller 1995; McWilliams et al. 1997; McWilliams and Restrepo 1999; Polton and Belcher 2007). The horizontally averaged momentum equations are

$$
\begin{aligned}
\frac{\partial\langle u\rangle}{\partial t}-f\langle v\rangle & =\frac{\partial \tau_{x z}}{\partial z} \quad \text { and } \\
\frac{\partial\langle v\rangle}{\partial t}+f\left(\langle u\rangle+u_{s}\right) & =\frac{\partial \tau_{y z}}{\partial z},
\end{aligned}
$$

where $\tau_{x z}$ and $\tau_{y z}$ are the total (resolved plus subgrid scale) along-wind and crosswind stress, respectively, and other symbols have been defined in section $2 \mathrm{~b}$. We introduce the vertically integrated transport

$$
\left(T_{x}, T_{y}, T_{s}\right)=\int_{-H}^{0}\left(\langle u\rangle,\langle v\rangle, u_{s}\right) d z
$$

where $H$ is the ocean depth, $T_{s}$ is the Stokes drift transport, and $T_{x}$ and $T_{y}$ are the along-wind and crosswind transports respectively. Integrating (A1) and (A2) over the water column results in the depth integrated Eulerian transport equations:

$$
\frac{d T_{x}}{d t}-f T_{y}=\tau \quad \text { and }
$$

$$
\frac{d T_{y}}{d t}+f\left(T_{x}+T_{s}\right)=0,
$$

where $\tau$ is the surface wind stress. For steady state (A4) implies crosswind Ekman transport, whereas the Stokes drift in (A5) induces an Eulerian return flow with the same magnitude but in opposite direction as the Stokes drift transport (McWilliams et al. 1997). Time-dependent solutions can be found by introducing $\mathbf{T}=T_{x}+i T_{y}$ with $i=\sqrt{-1}$, multiplying (A5) by $i$, and adding the resulting equation to (A4). This results in a first-order linear ordinary differential equation for $\mathbf{T}$,

$$
\frac{d \mathbf{T}}{d t}+\text { if } \mathbf{T}=\tau(t)-\text { if } T_{s}(t) .
$$

With the integrating factor, $\exp (i f t)$, the solution to (A6) is

$$
\mathbf{T}(t)=e^{-i f t}\left\{\int_{0}^{t} e^{i f t^{\prime}}\left[\tau\left(t^{\prime}\right)-i f T_{s}\left(t^{\prime}\right)\right] d t^{\prime}+\mathbf{T}_{0}\right\},
$$

where $\mathbf{T}_{0}$ denotes the initial transport. With $T_{s}=\mathbf{T}_{0}=0$ and $\tau=$ const (A7) simplifies to the familiar solution (see, e.g., Gill 1982, p. 322):

$$
\mathbf{T}(t)=-i \frac{\tau}{f}\left(1-e^{-i f t}\right),
$$

where the transport consists of an Ekman transport and inertial oscillation. Equation (A6) will always admit an inertial oscillation with amplitude and phase that satisfies the initial condition. Time-varying unidirectional wind stress and time-varying unidirectional Stokes drift will furthermore elicit rotating currents with along-wind and crosswind components. This is because any oscillating along-wind forcing can be decomposed into two counterrotating parts. The part rotating in the angular direction of the inertial currents is in resonance, while the forcing part that rotates in the opposite direction is off resonance. Therefore, the resulting current response to the two parts will not cancel, leading to a net rotating current with a period equal to the forcing period. This is seen by letting $\tau(t)=\tau_{0} \sin \left(\omega_{\tau} t\right)$ and $T_{s}(t)=T_{s 0} \sin \left(\omega_{s} t\right)$ with $\mathbf{T}_{0}=0$ so that the solution is

$$
\begin{aligned}
\mathbf{T}(t)= & \frac{i \tau_{0}}{2}\left(\frac{1}{f-\omega_{\tau}} e^{-i \omega_{\tau} t}-\frac{1}{f+\omega_{\tau}} e^{i \omega_{\tau} t}\right) \quad(\mathrm{I})+\frac{f T_{s 0}}{2}\left(\frac{1}{f-\omega_{s}} e^{-i \omega_{s} t}-\frac{1}{f+\omega_{s}} e^{i \omega_{s} t}\right) \\
& +\frac{1}{2}\left(\frac{i \tau_{0}}{f+\omega_{\tau}}-\frac{i \tau_{0}}{f-\omega_{\tau}}+\frac{f T_{s 0}}{f+\omega_{s}}-\frac{f T_{s 0}}{f-\omega_{s}}\right) e^{-i f t}
\end{aligned}
$$


The terms (I) and (II) represent the response due to wind and wave forcing with their angular frequencies of $\omega_{\tau}$ and $\omega_{s}$, respectively. For $\omega_{\tau}, \omega_{s}>0$, the first term within both (I) and (II) is resonantly rotating, while the second term within both (I) and (II) rotates off-resonance (full resonance is at $\omega_{\tau}, \omega_{s}=f$ ). The last term, (III), is the inertial current with amplitude and phase satisfying the boundary condition. During our simulation we therefore expect that the time varying unidirectional wind stress and Stokes drift will influence the transport response. The solution (A7) agrees well with the transport obtained from LES solutions (Fig. 1). These results provide an important additional test and further confidence in the complex LES solutions, on which our turbulence analysis is based on.

\section{APPENDIX B}

\section{Details on LES and Subgrid-Scale Model}

The governing LES momentum equation, adopted from McWilliams et al. (1997), is

$$
\begin{aligned}
\frac{\partial u_{i}}{\partial t} & +u_{j} \frac{\partial u_{i}}{\partial x_{j}}+\epsilon_{i k m} f_{k}\left(u_{m}+u_{s, m}\right) \\
= & -\frac{\partial \pi}{\partial x_{i}}+\frac{\rho}{\rho_{0}} g_{i}+\epsilon_{i k m} u_{s, k} \omega_{m}+\frac{\partial \tau_{i j}^{\mathrm{SGS}}}{\partial x_{j}} .
\end{aligned}
$$

Turbulent subgrid-scale fluxes are parameterized via an SGS eddy viscosity (e.g., $K_{M}$ for momentum),

$$
\tau_{i j}^{\mathrm{SGS}}=-K_{M}\left(\frac{\partial u_{i}}{\partial x_{j}}+\frac{\partial u_{j}}{\partial x_{i}}\right) .
$$

The coefficient $K_{M}$ depends on the SGS turbulent kinetic energy $e$ and an SGS length scale $l$, determined by the spatial resolution,

$$
K_{M}=l e^{1 / 2}
$$

in which $l=(\Delta x \Delta y \Delta z)^{1 / 3}$ if stratification is negative and $l=0.76 e^{1 / 2}(g \alpha \partial T / \partial z)^{-1 / 2}$ if stratification is positive (for details, see Moeng 1984). The SGS TKE, in turn, is determined from the prognostic equation (Deardorff 1973):

$$
\frac{\partial e}{\partial t}+u_{j} \frac{\partial e}{\partial x_{j}}=\tau_{i j}^{\mathrm{SGS}} \frac{\partial u_{i}}{\partial x_{j}}+g \alpha \tau_{T}^{\mathrm{SGS}}+\frac{\partial}{\partial x_{i}}\left(2 K_{M} \frac{\partial e}{\partial x_{i}}\right)-\epsilon,
$$

where $\tau_{T}^{\text {SGS }}$ is the SGS temperature flux and the turbulent dissipation rate $\epsilon$ is

$$
\epsilon=\frac{C e^{3 / 2}}{l}
$$

with

$$
C=0.19+0.51 l(\Delta x \Delta y \Delta z)^{-1 / 3} .
$$

Closer to the ocean surface the SGS model is modified for better correspondence with Monin-Obukhov similarity theory (Sullivan et al. 1994). In particular, this SGS model maintains the TKE formulation for the eddy viscosity but includes contributions from the mean flow and corrections of turbulent fluctuations near the surface.

At depths greater than $1 \mathrm{~m}$ (four or more grid points away from the air-sea interface), our simulations generally resolve at least $80 \%$ of the total (resolved plus SGS) turbulent kinetic energy. Therefore, our simulations can be considered "well resolved" (Pope 2008) for depths greater than $1 \mathrm{~m}$.

\section{REFERENCES}

Beare, R. J., and Coauthors, 2006: An intercomparison of largeeddy simulations of the stable boundary layer. Bound.-Layer Meteor., 118, 247-272, doi:10.1007/s10546-004-2820-6.

Craig, P. D., and M. L. Banner, 1994: Modeling wave-enhanced turbulence in the ocean surface layer. J. Phys. Oceanogr., 24, 2546-2559.

Craik, A. D. D., and S. Leibovich, 1976: A rational model for Langmuir circulations. J. Fluid Mech., 73, 401-426.

D'Asaro, E. A., and G. T. Dairiki, 1997: Turbulence intensity measurements in a wind-driven mixed layer. J. Phys. Oceanogr., 27, 2009-2022.

Deardorff, J. W., 1973: The use of subgrid transport equations in a three-dimensional model of atmospheric turbulence. J. Fluids Eng., 95, 429-438.

Drazin, P. G., and W. H. Reid, 2004: Hydrodynamic Stability. 2nd ed. Cambridge University Press, 605 pp.

Farmer, D., and M. Li, 1995: Patterns of bubble clouds organized by Langmuir circulation. J. Phys. Oceanogr., 25, 1426-1440.

Gargett, A. E., J. Wells, A. E. Tejada-Martinez, and C. E. Grosch, 2004: Langmuir supercells: A mechanism for sediment resuspension and transport in shallow seas. Science, 306, 1925-1928.

Gill, A. E., 1982: Atmosphere-Ocean Dynamics. Academic Press, $662 \mathrm{pp}$.

Gnanadesikan, A., and R. Weller, 1995: Structure and variability of the Ekman spiral in the presence of surface gravity waves. J. Phys. Oceanogr., 25, 3148-3171.

Grant, A. L. M., and S. E. Belcher, 2009: Characteristics of Langmuir turbulence in the ocean mixed layer. J. Phys. Oceanogr., 39, 1871-1887.

Harcourt, R. R., and E. A. D'Asaro, 2008: Large eddy simulation of Langmuir turbulence in pure wind seas. J. Phys. Oceanogr., 38, 1542-1562.

Ivey, G. N., K. B. Wintersand, and J. R. Koseff, 2008: Density stratification, turbulence, but how much mixing? Annu. Rev. Fluid Mech., 40, 169-184, doi:10.1146/annurev.fluid.39.050905.110314.

Kukulka, T., A. J. Plueddemann, J. H. Trowbridge, and P. P. Sullivan, 2009: Significance of Langmuir circulation in upper ocean mixing: Comparison of observations and simulations. Geophys. Res. Lett., 36, L10603, doi:10.1029/2009GL037620.

Langmuir, I., 1938: Surface motion of water induced by wind. Science, 87, 119-123, doi:10.1126/science.87.2250.119. 
Leibovich, S., 1983: The form and dynamics of Langmuir circulations. Annu. Rev. Fluid Mech., 15, 391-427.

— muir circulations. J. Fluid Mech., 102, 141-167.

Li, M., and C. Garrett, 1997: Mixed-layer deepening due to Langmuir circulation. J. Phys. Oceanogr., 27, 121-132.

,$- \ldots$, and E. Skyllingstad, 2005: A regime diagram for classifying turbulent large eddies in the upper ocean. Deep-Sea Res. I, 53, 259-278.

McWilliams, J. C., and J. Restrepo, 1999: The wave-driven ocean circulation. J. Phys. Oceanogr., 29, 2523-2540.

— , and P. P. Sullivan, 2000: Vertical mixing by Langmuir circulations. Spill Sci. Technol. Bull., 6, 225-237.

,-- , and C. H. Moeng, 1997: Langmuir turbulence in the ocean. J. Fluid Mech., 334, 1-30.

Melville, W. K., 1996: The role of surface-wave breaking in air-sea interaction. Annu. Rev. Fluid Mech., 28, 279-321.

Moeng, C.-H., 1984: A large-eddy-simulation model for the study of planetary boundary-layer turbulence. J. Atmos. Sci., 41, 2052-2062.

Plueddemann, A., J. Smith, D. Farmer, R. Weller, W. Crawford, R. Pinkel, S. Vagle, and A. Gnanadesikan, 1996: Structure and variability of Langmuir circulation during the surface waves processes program. J. Geophys. Res., 101 (C2), 3525-3543.

Polton, J. A., and S. E. Belcher, 2007: Langmuir turbulence and deeply penetrating jets in an unstratified mixed layer. J. Geophys. Res., 112, C09020, doi:10.1029/2007JC004205.

Pope, S. B., 2008: Turbulent Flows. 5th ed. Cambridge University Press, $771 \mathrm{pp}$.

Price, J., R. Weller, and R. Pinkel, 1986: Diurnal cycling: Observations and models of the upper ocean response to diurnal heating, cooling, and wind mixing. J. Geophys. Res., 91 (C7), 8411-8427.

Skyllingstad, E. D., and D. W. Denbo, 1995: An ocean large-eddy simulation of Langmuir circulations and convection in the surface mixed layer. J. Geophys. Res., 100 (C5), 8501-8522.

- W. D. Smyth, and G. B. Crawford, 2000: Resonant winddriven mixing in the ocean boundary layer. J. Phys. Oceanogr., 30, 1866-1890.

Smith, J. A., 1989: Doppler sonar and surface waves: Range and resolution. J. Atmos. Oceanic Technol., 9, 149-163.
1992: Observed growth of Langmuir circulation. J. Geophys. Res., 97 (C4), 5651-5664.

_ 1996: Observations of Langmuir circulation, waves, and the mixed layer. The Air Sea Interface: Radio and Acoustic Sensing, Turbulence, and Wave Dynamics, M. A. Donelan, W. H. Hui, and W. J. Plant, Eds., University of Toronto Press, 613-622.

_- 1998: Evolution of Langmuir circulation during a storm. J. Geophys. Res., 103, 12 649-12 668.

_ 1999: Doppler sonar observations of Langmuir circulation. Air-Sea Exchange: Physics, Chemistry and Dynamics, M. A. Donelan, W. H. Hui, and W. J. Plant, Eds., Kluwer Academic, 539-555.

__ and G. T. Bullard, 1995: Directional surface wave estimates from Doppler sonar data. J. Atmos. Oceanic Technol., 12, 617-632.

Smyth, W. D., E. D. Skyllingstad, G. B. Crawford, and H. Wijesekera, 2002: Nonlocal fluxes and Stokes drift effects in the K-profile parameterization. Ocean Dyn., 52, 104-115, doi:10.1007/s10236002-0012-9.

Sullivan, P. P., J. C. McWilliams, and J. C. Moeng, 1994: A subgrid-scale model for large-eddy simulation of planetary boundary-layer flows. Bound.-Layer Meteor., 71, 247-276.

,-- , and W. K. Melville, 2007: Surface gravity wave effects in the oceanic boundary layer: Large-eddy simulation with vortex force and stochastic breakers. J. Fluid Mech., 593, $405-452$.

Tejada-Martínez, A. E., and C. E. Grosch, 2007: Langmuir turbulence in shallow water. Part 2. Large-eddy simulation. J. Fluid Mech., 576, 63-108, doi:10.1017/S0022112006004587.

Terray, E., M. Donelan, Y. Agrawal, W. Drennan, K. Kahma, A. Williams, P. Hwang, and S. Kitaigorodskii, 1996: Estimates of kinetic energy dissipation under breaking waves. J. Phys. Oceanogr., 26, 792-807.

Thorpe, S. A., 2004: Langmuir circulation. Annu. Rev. Fluid Mech., 36, 55-79, doi:10.1146/annurev.fluid.36.052203.071431.

Weller, R. A., and J. F. Price, 1988: Langmuir circulation within the oceanic mixed layer. Deep-Sea Res., 35, 711-747.

Zedel, L., and D. Farmer, 1991: Organized structures in subsurface bubble clouds: Langmuir circulation in the open ocean. J. Geophys. Res., 96 (C5), 8889-8900. 